\title{
Mlit11 regulates the migration and neurite outgrowth of cortical projection neurons during development
}

Danielle Stanton-Turcotte ${ }^{1}$, Karolynn Hsu ${ }^{1}$, Samantha A. Moore ${ }^{1}$, Makiko Yamada,\#, James P. Fawcett $^{2}$, and Angelo Iulianella ${ }^{1} *$

${ }^{1}$ Department of Medical Neuroscience, and Brain Repair Centre, Faculty of Medicine, Dalhousie University. Life Science Research Institute, 1348 Summer Street, Halifax, Nova Scotia, Canada, B3H-4R2

${ }^{2}$ Departments of Phamacology, Surgery, and Brain Repair Centre, Faculty of Medicine, Dalhousie University. Life Science Research Institute, 1348 Summer Street, Halifax, Nova Scotia, Canada, B3H-4R2

\# Current address: Molecular Pharmacology Program, Memorial Sloan Kettering Cancer Center, 1275 York Avenue, ZRC-1863, New York, NY 10021.

* Correspondence: angelo.iulianella@ dal.ca

Running title: Mllt11 regulates development of callosal projection neurons.

Keywords: Mllt11/Af1q, callosal projection neurons, microtubules, neuritogenesis, axonogenesis, conditional transgenics, cortical dysplasia, neurodevelopmental disorders, gene expression.

Contributions: DST, KS, SM did the experiments, analysis and figures. DST and AI co-wrote the manuscript. JF advised with the GST-pull down experiments and assisted with the proteomic assays. AI assisted with imaging and supervised the project. 


\begin{abstract}
The formation of connections within the mammalian neocortex is highly regulated by both extracellular guidance mechanisms and intrinsic gene expression programs. There are generally two types of cortical projection neurons: those that project locally and interhemispherically, and those that project to sub-cerebral structures such as the thalamus, hindbrain, and spinal cord. The regulation of cortical projection morphologies is not yet fully understood at the molecular level. Here we report a role for Mllt11 (Myeloid/lymphoid or mixed-lineage leukemia; translocated to chromosome 11/All1 Fused Gene From Chromosome 1q) in the migration and neurite outgrowth of callosal projection neurons during brain formation. We show that Mllt11 expression is exclusive to developing neurons and is enriched in the developing cortical plate, particularly during the formation of the upper or superficial cortical layers. In cultured primary cortical neurons, Mllt11 is detected in varicosities and growth cones as well as the soma. Using conditional loss-of-function and gain-of-function analysis we show that Mllt11 is a required for neuritogenesis and proper migration of upper layer cortical projection neurons. Loss of Mllt11 in the superficial cortex leads to a severe reduction in fibres crossing the corpus callosum, a progressive loss in the maintenance of upper layer projection neuron gene expression, and dysplasia of dendritic arborisation patterns. Proteomic analysis revealed that Mllt11 associates with cytoskeletal components including stabilized microtubules consistent with a role in neuronal migration and neuritogenesis. Taken together, our findings support a role for Mllt11 in promoting the formation of mature projection neuron morphologies and connectivity in the cerebral cortex.
\end{abstract}




\section{INTRODUCTION}

The mammalian neocortex is a complex structure, underlying the capacity for executive function, sensory processing, emotion, motor output, and cognition. It is a laminated structure composed of six molecularly and morphologically distinct layers populated by a heterogeneous collection of excitatory pyramidal cortical projection neurons (CPNs) and inhibitory interneurons (INs). This anatomical organization emerges from the tight regulation of neurogenesis coupled with migration of newborn neurons to their final laminar position, where they acquire distinct molecular identities and morphological traits necessary to form functional circuitry within the brain. A fundamental question concerns how the migration of newborn neurons is controlled in the sequential generation of more superficial cortical layers and their subsequent connectivity patterns. While it is clear that the cytoskeleton plays a key role in neuronal translocation, how it impacts CPN subtype specific neurite morphogenesis and the maintenance of laminar transcriptional programs it not well understood.

The sorting of differentiating CPN subtypes into discrete, functional layers occurs as cells migrate radially from progenitor domains located along the ventricular zone (VZ) and adjacent subventricular zone (SVZ) along the apicobasal axis to invade the cortical plate (CP). Early in cortical development newly-born neurons migrate along radial glial fibres projecting from the basal surface of VZ progenitors to the pia via somal translocation (Miyata et al., 2001). Forces exerted on the cytoskeleton, including the microtubules that form the perinuclear cage, couple with motor proteins to enable appropriate mechanotransduction to push and pull the nucleus toward the pia until a neuron reaches its terminal location (Bellion et al., 2005; Tsai et al., 2007). As the somas of these cells reach the VZ, previously anchored apical junctional complexes are downregulated, releasing the soma from the VZ, and the trailing apical pole of the cells becomes the nascent axon projecting to form white matter tracts (Ayala et al., 2007; Marin and Rubenstein, 2003; Nadarajah et al., 2001; Sakakibara et al., 2014). Consequently, the intermediate zone (IZ) and CP become more densely populated with neurons and projections, and multiple modes of motility must be employed by later-born neurons to overcome the previously deposited neuronal layers. These events also rely on coordinated cytoskeletal reorganization as cells transiently acquire multipolarity to weave through the dense IZ and then reacquire bipolarity upon invasion of the CP via somal translocation (Sakakibara et al., 2014). The growth of axons 
during brain formation also involves dynamic cytoskeletal reorganization at the growth cone in response to external guidance cues or cell-cell contacts (Ayala et al., 2007; Hirokawa and Takemura, 2004).

Microtubule growth, retraction and stabilization are controlled by complex signaling from extracellular cues, and the coordination of these events are necessary for appropriate neuronal migration and neurite extension. The ability to couple developmental cues with construction or degradation of microtubule networks underlie the propensity of CPNs to form connections both intracortically and with other brain regions. As such, mutations of tubulin subtypes have been linked to neurodevelopmental disorders that affect cortical formation, stabilization, and projections. For instance, mutation of $\alpha$-tubulin isoforms cause lissencephaly and other gyrification defects, as well as reduction in white matter resulting in abnormalities of the corpus callosum (CC) and internal capsule (IC) (Bahi-Buisson et al., 2008; Jansen et al., 2011; Keays et al., 2007; Kumar et al., 2010; Poirier et al., 2007; Tian et al., 2010). $\beta$-tubulin mutations have been linked with cortical dysplasia, epilepsy, decreased neuronal density, enlarged ventricles, white matter thinning, and defects in migration and radial glial fibre extension due to their role in axonal growth, guidance, cell-cycle-dependent proliferation (Breuss et al., 2012; Breuss et al., 2017; Cushion et al., 2014; Ejaz et al., 2017; Hersheson et al., 2013; Jaglin et al., 2009; Jamuar et al., 2014; Ngo et al., 2014; Poirier et al., 2010; Rodan et al., 2017; Tischfield et al., 2010; Whitman et al., 2016). Mutation of $\gamma$-tubulin has also been shown to cause a wide range of phenotypes common to other tubulin isoforms due to its role in stabilizing growth and nucleation of microtubules (Brock et al., 2018; Draberova et al., 2017; Ivanova et al., 2019; Poirier et al., 2013; Yuba-Kubo et al., 2005).

Much like tubulin, mutations of microtubule associated proteins (MAPs) including stabilizing or nucleating proteins, motor proteins, and cargo-specific adapter proteins that govern trafficking of organelles and proteins to specific regions of a neuron, have also been linked with various forms of neurodevelopmental disorders (Moffat et al., 2015; Moon and Wynshaw-Boris, 2013). Mutations of these proteins can also affect neuronal development at the level of mitosis, axon guidance, disturbance of fasciculation of axonal tracts, or impaired migration. Mutations and aberrant localization of neurofilaments mediated by MT-based transport have also been 
linked with schizophrenia, bipolar disorder (BPD), and autism spectrum disorders (ASD) (Clark et al., 2007; English et al., 2009; Kristiansen et al., 2006; Pennington et al., 2008;

Sivagnanasundaram et al., 2007; Yao et al., 2021). A common thread between all mutations of tubulin isotypes and associated proteins is their propensity to give rise to phenotypes commonly associated with neurodevelopmental disorders through migration, cell fate specification, and axonal regulatory mechanisms required for functional connectivity within the cortex and between the cortex and other regions.

Although axonal regulatory mechanisms are conserved throughout the cortex, the targeting and wiring of each cortical layer is also dependent upon its unique molecular characteristics. For instance, each cortical layer expresses a unique program of transcription factors that provide the necessary molecular context crucial for synapses formation with the appropriate target regions of the brain. With the exception of the Cajal-Rietzus cells of layer 1 (L1), which are born first cells during corticogenesis and secrete Reelin to guide the radial migration of nascent neurons (Chai et al., 2009; Chai et al., 2016; Frotscher, 1998; Gil-Sanz et al., 2013), this process begins with Tbr1+ neurons which populate layer 6 (DL6) and project to the thalamus (Hevner et al., 2001; Thomson, 2010). Subsequently, the Bcl11b/Ctip2+ neurons of layer 5 (DL5) are born, which mostly connect to subcerebral regions (Arlotta et al., 2005). DL4 neurons, which receive input from the thalamus, can be identified by their co-expression of Foxp2 and Satb2 (Hisaoka et al., 2010; Leone et al., 2015; Lopez-Bendito and Molnar, 2003). The last cortical cell types generated are those of layers 2/3 (L2/3) in the superficial cortex, of which $80 \%$ project callosally and fewer than $20 \%$ project ipsilaterally within the cortex, identified by the expression of Cux1, Cux2 (Nieto et al., 2004; Zimmer et al., 2004), and Satb2 (Alcamo et al., 2008; Britanova et al., 2008; Leone et al., 2015).These transcription factors act in cross-repressive networks to regulate the organized chronological generation of cortical layers and functional diversity of projection neuron subtypes (Toma et al., 2014).

In an effort to identify regulators of neural migration and differentiation, we previously reported the expression of Mllt11/Af1q (Myeloid/lymphoid or mixed-lineage leukemia; translocated to chromosome 11/All1 Fused Gene From Chromosome 1q) in the developing central nervous system, including the cortical plate (Yamada et al., 2014). Mllt11 is a vertebrate- 
specific 90 amino acid protein that possesses a nuclear export signal but otherwise has poorly defined functional domains. Mllt11 was first identified in an infant with acute myelomonocytic leukemia carrying the $\mathrm{t}(1 ; 11)(\mathrm{q} 21 ; \mathrm{q} 23)$ translocation that creates an abnormal protein fused to Mll (Tse et al., 1995). Mllt11 may play a normal role in neural differentiation, as its transcription is down-regulated by RE1 silencing transcription factor, a key factor involved in regulating terminal differentiation of neurons (Hu et al., 2015). However, functional studies addressing its role in neural development are currently lacking. Here we report that Mllt11 is a neural-specific tubulin-associating protein and its conditional inactivation in the superficial layers of the mouse brain results in improper migration and formation of callosal projections. Additionally we show that Mllt11 loss led to dysplasia of the superficial cortex characterized by a severe loss of upper layer CPN dendritic morphologies resulting from neurite outgrowth defects. Interestingly, the inability to initiate and maintain functional projections in Mllt11 mutant brains was accompanied by a loss of upper cortical layer (UL)-specific transcriptional programs. On the other hand, Mllt11 overexpression promoted invasion of the cortical plate by differentiating neurons. Mechanistically, we provide evidence that Mllt11 interacts with stabilized microtubules, explaining the phenocopy of Mllt11 mutants with brain tubulinopathies (Bahi-Buisson et al., 2014). Altogether, these findings contribute significantly to our understanding of the genetic regulation of projection neuron development, dendritic complexity, and axonal connectivity.

\section{RESULTS}

\section{Conditional Knockout of Mllt11 from Upper Layer 2/3}

Mllt11 is expressed in developing neurons of the central nervous system, including the neocortex, but its role in cortical neurogenesis is unknown (Yamada et al, 2014). To investigate this, we generated mice carrying a null mutation in the Mllt11 gene using embryonic stem (ES) cell clones obtained from the mouse knockout consortium project (UCDavis KOMP repository, Mllt1 $1^{\text {tmla(KOMP)Mbp }}$. We used the targeted (unflipped) allele to visualize Mllt11 by $\beta$ galactosidase ( $\beta$-gal) staining due to the LacZ gene inserted into the Mllt11 locus (Fig. S1A, S4A). Initial Mllt11 locus activity begins in the pallial MZ at E12.5, showing stronger expression in the intermediate zone (IZ) of the fetal cortex at E14.5 coinciding with the birth of basal progenitors fated to become UL2/3 CPNs, intensifying in the superficial cortical layers by E16.5E18.5 (Fig S4A). This confirms our previous report of Mllt11 mRNA and protein localization in 
these regions (Yamada et al., 2014). Subsequent in situ hybridization revealed that cortical expression is maintained until postnatal day $21(\mathrm{P} 21)$ when it begins to taper to levels indistinguishable from background at P28 (Fig. S1B-C). Mllt11 transcript levels decreased but remained detectable in the hippocampus on through P28 (Fig. S1C).

Given the temporal and spatial overlap between Mllt11 expression and neurogenic regions of the cerebrum, we set out to investigate the role of Mllt11 in the development cerebral cortex, and specifically in the formation of the upper layer (UL) $2 / 3$ callosally projecting CPNs that bridge communication interhemispherically (Fame et al., 2011) where Mllt11 expression appeared highest during corticogenesis (Fig. S4A). We utilized the targeted Mllt11 null allele to generate a conditional knockout (cKO) Mllt11 allele in which the entire coding sequence in Exon 2 is flanked by loxP sites. To avoid non-specific effects of the selection cassette, mice encoding germline expressing flp recombinase were crossed to mice harbouring the Mllt11 targeted allele, leaving only loxP sites used to excise the entire protein-coding exon to create a conditional allele. Subsequently, in order to inactivate the Mllt11 in UL2/3 of the cerebral cortex, we crossed the Mllt11 $1^{\text {Flox/Flox }}$ mice to the Cux2iresCre strain along with the Ai9 TdTomato reporter mice to visualize recombined neurons, creating Mllt11 conditional knockout mutants (cKO). Cux2 is highly expressed in developing UL2/3 projections neurons, and mosaically expressed in the SVZ in the pallial cortex (Cubelos et al., 2008a; Cubelos et al., 2008b; Nieto et al., 2004; Zimmer et al., 2004). The resulting Cux2iresCre-driven Mllt11 cKO mice should lack Mllt11 expression in UL of the neonatal cortex. We confirmed Mllt11 loss in UL CPNs qPCR of E18.5 cortices (Fig. S2A-B) as well as by ISH at P7 (Fig. S2C-D). Due to our genetic strategy, all cells exhibiting Mllt11 loss were labeled by TdTomato fluorescence. TdTomato labeling was observed mostly in UL 2/3 of the cortex (Fig. S3A) and commissural tracts at P7 (cc; Fig. S3B), confirming that Cremedicated excision was specific to the $C u x 2$ expression domain.

\section{Loss of Mllt11 from Upper Layer 2/3 Leads to Progressive Decrease in Cortical Thickness}

Brain and body weight were not found to differ significantly between Mllt11 cKO mutants and controls at E18.5, though there was a very slight trend toward decreased brain weight in the cKOs (Fig. S4B-D). Mutants displayed a thinning of the cortex visible in the cortical plate (CP) and white matter (WM) beneath it (Fig. S4E; Fig. 4A, D). To explore the origin of this cortical thinning, we examined the morphology of the cortex beginning at E14.5 
(Fig. S4F) when UL CPNs are born and begin to express Cux2 (Cubelos et al., 2008a; Zimmer et al., 2004). The thinning of the cortex was observed beginning at E16.5 (Fig. S4G), increasing in severity by E18.5 (Fig. S4H). We noted a non-significant trend toward fewer total DAPI+ cells in the Mllt11 mutant cortex (Fig.S4H). However, we report significant changes in the distribution of DAPI+ nuclei in deeper bins of the Mllt11 cKOs, with a larger proportion of DAPI+ nuclei localized apically in E18.5 mutants relative to controls (Fig. S4F-H). We also evaluated whether programmed cell death could account for the cortical thinning, but did not find any cells positive for the apoptotic marker cleaved caspase 3 (CC3) in the Mllt11 neonatal cortex (Fig S5A-B), while retrosplenial regions, which typically show enhanced apoptosis, showed comparable levels of CC3+ staining in controls and mutants (Fig. S5C).

Mllt11 expression was restricted to the developing cortical plate and not in the apical progenitor regions (VZ) of the cortex (Fig. S4A). To rule out potential cell non-autonomous contributions from neural progenitors to the Mllt11 mutant cortical phenotype we quantified cells positive for progenitor markers from E14.5-18.5, corresponding to the period of UL neurogenesis. Pax6, a radial glial cell (RGC) marker (Gotz et al., 1998), was unaltered at all observed time points (Fig. S6A-C). Sox2, a less restrictive marker for neural progenitors and quiescent RGCs (D'Amour and Gage, 2003; Ellis et al., 2004), also exhibited no significant differences in expression (Fig. S6D-F). Tbr2+ intermediate (basal) progenitors, which give rise to UL 2/3 CPNs, did not differ markedly between control and Mllt11 cKO groups, but displayed fewer cells within deep (non-progenitor) regions of the mutant cortex at E18.5, suggesting alterations in the formation and/or migration of nascent neurons from the SVZ (Fig. S6G-I).

\section{Mllt11 is Required for the Maintenance of UL CPN Molecular Identity}

The transcriptional de-repression loop that specifies cell types in discrete cortical laminae coincides with the birth and migration of projection neurons (Kumamoto et al., 2013; Toma et al., 2014). Thus a loss of Mllt11 in UL progenitors can potentially affect neuronal birth, migration, and/or specification. We therefore investigated whether Mllt11 loss had any role in regulating the molecular identity of UL CPNs. Expression of Satb2 in CPNs of L2-4 exhibited a decrease at E18.5 (Fig. 1A) as did CDP/Cux1, another marker of UL CPNs (Fig. 1B). Investigation at earlier developmental time points revealed that this phenotype was progressive, beginning at E16.5 and increasing in severity by E18.5 (Fig. S7A-D). While the extent of CDP/Cux1 and Satb2 staining 
displayed variability across individual mutants, there was a consistent decrease in expression levels and downward shift in expression domain in all Mllt11 cKOs. TdTomato labelling was used to identify $\mathrm{Cux} 2+$ cell labeling in intermediate progenitors that give rise to UL2/3 CPNs, and while reporter levels were found to be unaltered at all time points, there was a consistent downward shift in expression domain at E18.5 (Fig, 1C, Fig. S7E-F). Collectively, these findings suggested that Mllt11 loss in nascent UL neurons did not affect their neurogenesis, or the activation of UL gene expression programs, but may have affected their migration to contribute to $\mathrm{L} 2 / 3$ formation.

The cortical de-repression loop functions on a cellular level by alternately repressing DL and UL fate (Toma et al., 2014) so we wanted to determine if the loss of UL CPN fate was coupled with a complementary upregulation of DL CPN fates in these cells. We examined the localization of DL6- and DL5-specific markers Tbr1 (Fig. 1D) and Ctip2 (Fig. 1E) respectively, which both exhibited similar numbers and staining patterns between Mllt11 cKOs and controls at all time points (Fig. S7G-J). However, the mutants displayed a downward shift of the expression domains of both markers consistent with the decreased cortical thickness and UL formation (Fig. 1D-E). To determine whether the loss of CPN subtype-specific markers was indicative of loss of neuronal identity, we examined the localization of NeuN, a marker for maturing neurons (Mullen et al., 1992) and found no alterations in Mllt11 cKOs (Fig. S8A-B). No changes in GFAP staining, a marker of radial glial fibres and astrocytes (Bignami and Dahl, 1977; Zhang, 2001) was apparent, demonstrating that these Mllt11 mutant cortical cells were not experiencing a shift from neuronal to glial identity (Fig. S8C). This is consistent with the neuronally restricted expression of Mllt11 (Fig. S1C, S4A) (Yamada et al., 2014).

Given that the Mllt11 mutants failed to maintain UL-specific transcription factors over time, we wanted to understand whether the cortical de-repression loop and layer-specific celltype specification were de-coupled, such that CPNs born at the time of UL CPN birth could instead mis-express a DL-specific transcriptional regulator. Pregnant dams were injected with EdU at E14 or E16 to label UL neurogenesis and embryos were harvested at E18.5 to examine the co-localization of the DL CPN marker Ctip2 with EdU. The proportion of Ctip2+ CPNs born at E14 did not differ significantly between controls and cKOs with the exception of the overall downward shift in the distribution of Ctip2+/EdU+ cells in the cKOs (Fig. S9A-a'). Embryos pulsed with EdU at E16 and harvested at E18.5 displayed the same trend, exhibiting a downward 
shift but no significant differences in overall proportions of Ctip2+/EdU+ co-labeled cells (Fig. S9B-b'). Taken together, these data suggest that UL CPNs are not experiencing a fate change to DL CPNs, but initiate expression of proper layer-specific transcription factors which they fail to maintain over time.

\section{Mllt11 is Required for the Migration of CPN Progenitors}

Given the thinning of the cortex and downward shift of cortical layer markers throughout development, we hypothesized that Mllt11 may regulate $\mathrm{CPN}$ migration into the $\mathrm{CP}$. To explore this, we pulsed pregnant dams with EdU to label UL CPNs at E14 or E16 and observed the localization of EdU at E18.5. CPNs labeled with EdU at E14 exhibited a severe downward shift of roughly $100 \mu \mathrm{m}$, consistent with the decrease in cortical thickness (Fig. 2A). A greater proportion of E14-born CPNs remained restricted to the deeper bins of Mllt11 cKO cortices relative to controls (Fig. 2A). As CPNs labeled with EdU at E16 were still in transit by E18, with only a few control CPNs reaching the outermost layers of the cortex, the discrepancy in UL cell migration was less pronounced in cKOs (Fig. 2B). To examine whether Mllt11 loss affected the neurogenesis of UL neurons, we pulsed dams with EdU for 2 hours at E14, which efficiently labeled nuclei within the basal progenitor region (Fig. 2C). We observed no difference in the numbers or distribution of EdU+ nuclei in Mllt11 mutants vs. control cortices after a short pulse at E14 (Fig. 2C). We repeated this short pulse at E18, when UL neurogenesis is nearly complete and noted no differences in EdU+ cells in the superficial cortex of Mllt11 mutant vs. control brains (Fig. 2D). We did observe a slight increase of EdU+ cells in the deepest $100 \mu \mathrm{m}$ bin of the Mllt11 mutant cortex relative to controls, possibly reflecting aberrant migration of later born neurons (Fig. 2D). Overall, the EdU birth dating analysis suggested that Mllt11 primarily regulates the migration, but not neurogenesis, of UL CPNs into the cortical plate.

A more conclusive test for a role of Mllt11 in the migration of CPN progenitors into the cortical plate would be to overexpress it in the developing fetal brains. We therefore used in utero electroporation to express a bicistronic vector containing Mllt11 and GFP cDNAs, or control GFP vector alone, in wild type mouse embryonic forebrains. We performed electroporations in anesthetised pregnant dams at E13.5, by injecting a DNA solution containing expression vectors unilaterally into forebrain ventricles, and analyzed the resulting fetal brains 2 days after at E15.5, when neurons are migrating into the cortical plate. At E15.5, CPNs overexpressing Mllt11 
primarily colocalized with preplate marker Tbr1 (Fig. 3A), while GFP-expressing control CPNs localized more closely with Tbr2+ basal progenitors near the IZ (Fig. 3B-D), demonstrating Mllt11 overexpression is sufficient to promote CPN migration into the developing cortical plate. Taken together, our loss and gain-of-function analyses strongly support a role for Mllt11 in promoting nascent UL neuronal migration in the developing cortical plate.

The migration of CPN progenitors is facilitated by a meshwork of glial fibres which extend from the VZ towards the pial surface (Campbell and Gotz, 2002; Hartfuss et al., 2001; Miyata et al., 2001). These fibres act as tracks for extending neurites guiding neuronal translocation, contributing to the meshwork laid down by earlier born progenitors. As such, the orientation of Nestin+ fibres can indicate the direction of migration into the CP (Gotz et al., 1998; Nadarajah et al., 2001). We wanted to rule out any possible cell non-autonomous effects of Mllt11 loss on the migrating scaffold in the developing cortex by carefully analyzing the Nestin+ glial scaffold of the cortex. We found no significant alterations in orientation, density, and dispersion of RGC fibres in Mllt11 cKOs during cortical development (Fig. S10), but did note a non-significant trend toward a greater dispersion of the fibres in cKOs relative to controls at E18.5 (Fig. S10L). Guidance of migratory CPNs is dependent on the earliest born Cajal-Rietzus (CR) cells of L1, which secrete Reelin to attract and halt neuronal migration at the pial interface (Hirota and Nakajima, 2017; Tueting et al., 1999). However, there were no differences in the expression of CR markers p73 or Reelin at E14.5 (Fig. S11A) or E18.5 (Fig. S11B) following Mllt1 1 loss in UL progenitors and nascent neurons. Altogether, these findings demonstrate that the aberrant migration of UL CPNs in Mllt11 mutants was not caused by alterations in the development of Reelin-secreting CR cells, nor abnormalities in radial glial fibre scaffold. Rather, Mllt11 seems to be required cell autonomously to promote invasiveness of nascent neurons into the developing cortical plate.

\section{Mllt11 Loss Leads to Reduced Callosal Crossing Fibres and Thinning of WM tracts}

The migratory defects alone may be insufficient to explain the decreased cortical thickness of Mllt11 cKO brains at E18.5 (Fig.S4). Given the reduction in UL CPNs (Fig. 1) would largely affect interhemispheric projections (Aboitiz and Montiel, 2003; Alcamo et al., 2008; Fame et al., 2011), we next examined whether Mllt11 loss impacted formation of projection fibres contributing to the cortical white matter (WM) tracts by staining for NF in 
coronal brain slices. We observed notable decreases in the thickness of NF+ fibres in the WM tract and in the proportion of the cortex containing WM between E16.5 (control=235 $\mu \mathrm{m} \pm 49.68$, $\mathrm{cKO}=172 \mu \mathrm{m} \pm 17.06$; Fig. 4A-C) and E18.5 (control=235.1 $\mu \mathrm{m} \pm 12.58, \mathrm{cKO}=200.8 \mu \mathrm{m} \pm$ 15.66; Fig. 4D-F) following Mllt11 loss. Importantly, while NF+ fibre staining in the corpus callosum was greatly reduced in Mllt11 cKOs at E18.5, NF+ fibres in the internal capsule, reflecting corticothalamic projections, remained unchanged in the mutant brains (ic; Fig. 4G). Taken together, our findings revealed a critical role for Mllt11 in UL CPN neurite morphology and axonogenesis of callosal fibres connecting the developing cerebral hemispheres.

The reduction of NF+ fibres crossing the corpus callosum in mutant brains suggested that Mllt11 loss impacted the ability of UL2/3 CPNs to extend fibres across the telencephalic midline. To address this, we injected the lipophilic fluorescent dye DiI into the WM of cortical slices at E18.5 and allowed it to diffuse through the tissue for 2 weeks before visualization. DiI labeling was not detectable in the callosal fibres of Mllt11 cKOs, while controls exhibited clearly labeled crossing fibres (Fig. 4H). In contrast, the corticothalamic projections of the internal capsule were intact and traced by the DiI in both controls and cKOs, demonstrating that corticothalamic projections from DL 6 of the cortex were unaffected by the Cux2iresCre driven Mllt1 1cKO strategy (ic; Fig. 4H). Altogether, our findings demonstrated that Mllt11 loss in UL neurons specifically impacted the development of callosal projections.

\section{Mlt11 Interacts with Cytoskeletal Proteins}

Our birthdating analysis suggested that the migratory defects observed in Mllt11 cKOs are likely independent of the maintenance of UL transcriptional programs. To identify potential pathways through which Mllt11 might be exerting its effects on CPN migration, we performed a GST pulldown assay to identify interacting proteins using lysate from E18.5 brains. Potential Mllt11-interacting proteins are listed in Table 1 and include multiple $\alpha$ and $\beta$ tubulin isoforms (> $70 \%$ coverage) as well several atypical myosins, such as Myosin 5a (36\%), Myosin 9 (24\%) and Myosin 10. A potential association between Mllt11 and tubulin is consistent with a recent whole cell proteomic study, which identified Mllt11 as a likely interactor of both $\alpha$ - and $\beta$-tubulins (Go et al., 2021). Given that acetylation of $\alpha$-tubulin is associated with stabilized microtubules and is crucial for outgrowth of stable neurites, we decided to focus on validating interactions between Mllt11and acetylated $\alpha$-tubulin by immunoprecitiation analysis. We overexpressed a myc-tagged 
Mllt11 in Hek293 cells and pulled down acetylated- $\alpha$-tubulin (Fig. 5A). Probing the blots with anti-Mllt11 antibodies confirmed this interaction was dependent on heterologous Mllt11 expression in the cells (Fig. 5B). We also confirmed an association between Mllt11 and Myosin 10 (data not shown). Thus, we validated an association between Mllt11 and stabilized tubulin isoforms.

To determine whether Mllt11 regulates tubulin expression, western blots were performed on lysates from Mllt11 cKO and control E18.5 cortices. Tubb3 (a $\beta$-tubulin isoform) and acetylated $\alpha$-tubulin levels appeared comparable between E18.5 Mllt11 mutant and controls cortices (N=3, Fig. 5C-D). Interestingly, there was a trend towards an increase in the ratio of acetylated tubulin to Tubb3 in Mllt11 cKOs, although it was not statistically significant (Fig. 5E). This suggested the possibility that Mllt11 loss affected the formation of stabilized microtubules.

To evaluate whether Mllt11 localized to growing neurites, we used immunocytochemistry to probe the sub-cellular distribution of Mllt11, Tubb3, and acetylated $\alpha$-tubulin in cultures of primary fetal cortical neurons. Cortical neurons were extracted from wild type embryos at E18.5 and cultured for 24 hours or 1 week, then immunostained to reveal the localization of Tubb3, acetylated $\alpha$-tubulin, and Mllt11. After 24 hours in vitro, corresponding to the extension of a primary neurite, Mllt11/Tubb3 co-localized in discrete punctate patterns in varicosities along the distal portion of the developing neurite, as well as in the growth cone (Fig 5F-f'). Limited overlap was seen in the soma (Fig. 5F). After 1 week in culture, neurons displayed a more elaborate neurite arborisation pattern and acetylated $\alpha$-tubulin and Mllt11 were both detected along proximal portion of neurite (Fig. 5G). In summary, Mllt11 expression displayed a high degree of overlap with acetylated $\alpha$ - and $\beta$-tubulin isoforms differentially over neurite development, consistent with a role in neurite development. This is in agreement with a study identifying possible microtubule interactions with Mllt11 (Go et al., 2021) 
Table 1. Potential Mllt11 interaction targets from GST pull downs in E18.5 whole brain lysates.

\begin{tabular}{|c|c|c|c|c|c|c|c|c|c|}
\hline Band & Accession & Description & $\begin{array}{l}\text { Sum PEP } \\
\text { Score } \\
\end{array}$ & $\begin{array}{c}\text { Coverage } \\
{[\%]} \\
\end{array}$ & \# Peptides & $\begin{array}{c}\# \\
\text { PSMs } \\
\end{array}$ & $\begin{array}{r}\# \\
\text { AAs } \\
\end{array}$ & $\begin{array}{c}\text { MW } \\
{[\mathrm{kDa}]} \\
\end{array}$ & Reference \\
\hline 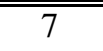 & D3Z4J3 & "Unconventional myosin-Va (Myo5a) & 201.776 & 36 & 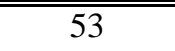 & 240 & 1855 & 215.4 & \\
\hline 7 & Q8VDD5 & Myosin-9 (Myh9) & 181.542 & 24 & 36 & 142 & 1960 & 226.2 & \\
\hline 6 & Q3UH59 & Myosin-10 (Myh10) & 31.272 & 8 & 10 & 22 & 2013 & 233.3 & \\
\hline 5 & P63017 & $\begin{array}{l}\text { Heat shock cognate } 71 \mathrm{kDa} \text { protein OS=Mus musculus } \\
\mathrm{OX}=10090 \mathrm{GN}=\mathrm{Hspa} 8 \mathrm{PE}=1 \mathrm{SV}=1\end{array}$ & 199.247 & 59 & 32 & 303 & 646 & 70.8 & Li et al., 2014 \\
\hline 5 & P38647 & Stress-70 protein, mitochondrial (Hspa9) & 20.395 & 12 & 6 & 12 & 679 & 73.4 & \\
\hline 5 & P20029 & Endoplasmic reticulum chaperone BiP (Hspa5) & 23.74 & 10 & 5 & 50 & 655 & 72.4 & \\
\hline 5 & Q3THK7 & GMP synthase [glutamine-hydrolyzing] (Gmps) & 8.67 & 5 & 3 & 6 & 693 & 76.7 & \\
\hline 5 & $\mathrm{O} 08553$ & $\begin{array}{l}\text { Dihydropyrimidinase-related protein } 2 \mathrm{OS}=\text { Mus musculus } \\
\mathrm{OX}=10090 \mathrm{GN}=\text { Dpysl2 } \mathrm{PE}=1 \mathrm{SV}=2\end{array}$ & 12.297 & 15 & 4 & 7 & 572 & 62.2 & \\
\hline 4 & Q9CWF2 & Tubulin beta-2B chain (Tubb2b) & 381.396 & 74 & 29 & 1338 & 445 & 49.9 & \\
\hline 4 & Q7TMM9 & Tubulin beta-2A chain (Tubb2a) & 380.755 & 74 & 29 & 1340 & 445 & 49.9 & \\
\hline 4 & P99024 & Tubulin beta-5 chain (Tubb5) & 369.155 & 74 & 29 & 1232 & 444 & 49.6 & \\
\hline 4 & $\mathrm{P} 68372$ & Tubulin beta-4B chain (Tubb4b) & 365.578 & 74 & 29 & 1153 & 445 & 49.8 & \\
\hline 4 & Q9ERD7 & Tubulin beta-3 chain (Tubb3) & 347.246 & 72 & 28 & 970 & 450 & 50.4 & \\
\hline 4 & Q9D6F9 & Tubulin beta-4A chain (Tubb4a) & 324.979 & 73 & 27 & 993 & 444 & 49.6 & Go et al., 2021 \\
\hline 4 & P68369 & Tubulin alpha-1A chain (Tuba1a) & 255.603 & 66 & 27 & 557 & 451 & 50.1 & Go et al., 2021 \\
\hline 4 & $\mathrm{P} 05213$ & Tubulin alpha-1B chain (Tuba1b) & 241.479 & 66 & 26 & 519 & 451 & 50.1 & \\
\hline 4 & Q922F4 & Tubulin beta-6 chain (Tubb6) & 176.166 & 40 & 17 & 444 & 447 & 50.1 & \\
\hline 4 & P10126 & Elongation factor 1-alpha 1 (Eef1a1) & 74.451 & 37 & 10 & 62 & 462 & 50.1 & \\
\hline 4 & A2AQ07 & Tubulin beta-1 chain (Tubb1) & 54.274 & 13 & 7 & 202 & 451 & 50.4 & \\
\hline 4 & Q9D8N0 & Elongation factor 1-gamma (Eef1g) & 27.863 & 20 & 6 & 14 & 437 & 50 & \\
\hline 4 & Q3UX10 & Tubulin alpha chain-like 3 (Tubal3) & 30.405 & 8 & 4 & 89 & 446 & 50 & \\
\hline 3 & Q9CWF2 & Tubulin beta-2B chain (Tubb2b) & 239.301 & 71 & 24 & 304 & 445 & 49.9 & \\
\hline 3 & P99024 & Tubulin beta-5 chain (Tubb5) & 214.778 & 71 & 24 & 324 & 444 & 49.6 & \\
\hline 3 & Q9D8N0 & Elongation factor 1-gamma (Eef1g) & 193.321 & 65 & 23 & 324 & 437 & 50 & \\
\hline 3 & P10126 & Elongation factor 1-alpha 1 (Eef1a1) & 202.141 & 56 & 21 & 542 & 462 & 50.1 & \\
\hline 3 & Q9ERD7 & Tubulin beta-3 chain (Tubb3) & 158.874 & 64 & 21 & 199 & 450 & 50.4 & \\
\hline 3 & P68369 & Tubulin alpha-1A chain (Tuba1a) & 188.056 & 64 & 20 & 177 & 451 & 50.1 & Go et al., 2021 \\
\hline 3 & P05213 & Tubulin alpha-1B chain (Tuba1b) & 178.724 & 64 & 20 & 169 & 451 & 50.1 & \\
\hline 3 & Q9D6F9 & Tubulin beta-4A chain (Tubb4a) & 170.961 & 54 & 19 & 253 & 444 & 49.6 & Go et al., 2021 \\
\hline
\end{tabular}




\begin{tabular}{|c|c|c|c|c|c|c|c|c|}
\hline 3 & P62631 & Elongation factor 1-alpha 2 (Eef1a2) & 50.614 & 28 & 9 & 259 & 463 & 50.4 \\
\hline 3 & Q8BFR5 & Elongation factor Tu, mitochondrial (Tufm) & 39.656 & 32 & 9 & 28 & 452 & 49.5 \\
\hline 3 & P60710 & Actin, cytoplasmic 1 (Actb) & 41.856 & 31 & 7 & 24 & 375 & 41.7 \\
\hline 3 & P17182 & Alpha-enolase (Eno1) & 27.891 & 24 & 7 & 12 & 434 & 47.1 \\
\hline 3 & P62737 & Actin, aortic smooth muscle (Acta2) & 24.601 & 16 & 5 & 16 & 377 & 42 \\
\hline 3 & Q3UX10 & Tubulin alpha chain-like 3 (Tubal3) & 19.056 & 8 & 4 & 36 & 446 & 50 \\
\hline 3 & P10637-3 & Isoform Tau-B of Microtubule-associated protein tau (Mapt) & 14.659 & 14 & 4 & 17 & 364 & 38.2 \\
\hline 2 & $\begin{array}{l}\text { Custom- } \\
\text { P97783 }\end{array}$ & Protein AF1q (Mllt11) & 298.867 & 89 & 35 & 1363 & 320 & 36.7 \\
\hline 2 & $\mathrm{P} 48758$ & Carbonyl reductase [NADPH] 1 (Cbr1) & 185.195 & 77 & 21 & 576 & 277 & 30.6 \\
\hline 2 & P62908 & 40S ribosomal protein S3 (Rps3) & 85.426 & 70 & 17 & 171 & 243 & 26.7 \\
\hline 2 & $\mathrm{P} 68040$ & Receptor of activated protein C kinase 1 (Rack1) & 34.523 & 25 & 7 & 20 & 317 & 35.1 \\
\hline 2 & O70251 & Elongation factor 1-beta (Eef1b) & 16.545 & 24 & 4 & 9 & 225 & 24.7 \\
\hline 2 & Q9D819 & Inorganic pyrophosphatase (Ppa1) & 10.238 & 15 & 3 & 6 & 289 & 32.6 \\
\hline 2 & $\begin{array}{l}\text { Q80XN0 } \\
\end{array}$ & $\begin{array}{l}\text { D-beta-hydroxybutyrate dehydrogenase, mitochondrial } \\
\text { (Bdh1) }\end{array}$ & 8.871 & 9 & 2 & 4 & 343 & 38.3 \\
\hline 1 & P97783 & Protein AF1q (Mllt11) & 48.499 & 92 & 6 & 111 & 90 & 10 \\
\hline
\end{tabular}




\section{Mllt11 is Required for Neurite Outgrowth and Extension}

The relationship between stabilized and dynamic forms of tubulin has been shown to regulate the migratory potential of neurons and neurite extension due to the cycling of microtubule severing during outgrowth (Lin and Smith, 2015; Sudo and Baas, 2010; Wei et al., 2018). If Mllt11 loss affected the relative amounts of stabilized microtubules, we would expect to see significant changes in neurite morphogenesis of UL neurons. We therefore evaluated the morphology of cultured primary cortical neurons from Mllt11 cKOs. We took advantage of the $\mathrm{Ai} 9$ tdTomato+ reporter introduced into the Cux2iresCre/+; Mllt1 $1^{\text {Flox/+ }}$ conditional mouse to efficiently label and isolate UL primary neurons in culture. After 24 hours in vitro, the length of MAP2+ primary neurites was reduced in cKOs compared to controls (control=83.78 $\mu \mathrm{m} \pm 23.17$, $\mathrm{cKO}=73.62 \mu \mathrm{m} \pm 24.52, \mathrm{~N}=150$; Fig. $6 \mathrm{~A}-\mathrm{B}$ ), as was the total length of all neurites (control=127.4 $\mu \mathrm{m} \pm 56.11, \mathrm{cKO}=92.34 \mu \mathrm{m} \pm 28.15, \mathrm{~N}=150$; Fig. $6 \mathrm{C}$ ). Moreover, the proportion of total length of extended neurites represented by the primary neurite at 24 hours was slightly increased in cKOs (control=.72 $\pm .21, \mathrm{cKO}=.81 \pm .18, \mathrm{~N}=150$; Fig. 6D). However, after 1 week of culture in vitro, the total neurite length was drastically reduced in Mllt11 cKO CPNs (control=1415 $\mu \mathrm{m} \pm$ 1112, $\mathrm{cKO}=595.9 \mu \mathrm{m} \pm 597.2, \mathrm{~N}=150$; Fig. 6E). Since Mllt11 loss progressively attenuated primary neurite outgrowth in cultured primary UL CPNs, we next evaluated a requirement for Mllt11 in the elaboration of dendritic arborisation patterns in cultured CPNs. An advantage of using cultured neurons is that they are free of apical and basal contacts, allowing their neurites to radiate uniformly from the soma. This allows for the evaluation of dendritic complexity by Sholl analysis. Mllt11 mutant primary neurons displayed much less complexity in the branching of their neurites (Fig. 6F-G), thus Mllt11 loss greatly impacted the arborisation patterns of cultured UL CPNs.

Given the vast array of morphological growth and pruning in early perinatal development, we next evaluated the requirement of Mllt11 in the formation of dendritic arborisation morphology characteristic of UL CNs in vivo at a time when mature arborisation patterns have fully developed. Golgi staining was performed on Mllt11 cKO and control brains harvested at P28 to capture mature CPN morphologies (Fig. 7). Importantly, we observed a severe decrease total neurite length in UL2/3 CPNs in cKOs relative to controls (control $=1176.62 \mu \mathrm{m} \pm 164.73$, $\mathrm{cKO}=563.85 \mu \mathrm{m} \pm 106.1$, Fig. 7A-D). The extent of decrease of neurite aborisation in vivo was similar to that observed in cultured primary neurons. Sholl analysis revealed that the overall 
neuronal morphology was also much less complex with less elaborately-branched neurites in Mllt11 cKOs (Fig. 7E). Taken together, these findings confirmed a critical requirement of Mllt11 in the growth and elaboration of neurites during the development of mature superficial CPN morphologies and projection phenotypes.

\section{DISCUSSION}

We described the role of a novel type of a vertebrate-specific neuronal restricted protein, Mllt11, which acts as a regulator of neurite extension and migration of superficial cortical projection neurons. Using the Cux2iresCre driver to excise Mllt11 in most developing UL cortical neurons, we revealed that Mllt11 loss led to decreased cortical white matter tracts and callosal fibres crossing the forebrain midline, as well as an inability to extend and maintain mature, arborescent dendrites. Nestin+ and GFAP+ fibres and progenitor markers were largely intact in the absence of Mllt11, consistent with the expression of Mllt11 in the developing cortical plate, but not in VZ progenitors. Furthermore, Mllt11 conditional mutants did not affect the formation of layer 1 Reelin+ cells, which guides the migration of cortical neurons and formation of fibre scaffolds, suggesting instead that Mllt11 loss acted cell autonomously to affect the radial migration of CPN progenitors into the cortical plate. The inefficient migration of UL CPNs in Mllt11 mutant brains likely reflected a role for Mllt11 in regulating the cytoskeleton, as is associated efficiently with stabilized microtubules. Neurons lacking Mllt11 displayed deficits in development of arborescent neurites both in vivo and in vitro. This neurite extension deficit in the mutants was further confirmed by the decrease in neurofilament and DiI staining of callosal fibres, and thinning of white matter tracts of the cortex, demonstrating that Mllt11 is a regulator of neurite outgrowth and maintenance required for forebrain connectivity.

Cortical thinning and increased ventricular lumen surface area are common phenotypes associated with neurodevelopmental disorders such as autism, suggesting cytoskeletal dysregulation may underlie the occurrence of a subset of these disorders (Bahi-Buisson et al., 2014; Li et al., 2019; Mensen et al., 2017). We now show that Mllt11 interacts with tubulin isoforms, confirming findings by others in a recent proteomic screen (Go et al., 2021), which provide insight into the mechanistic basis of the Mllt11 mutant phenotype. The microtubule cytoskeleton generates polarized force and provides a functional railway along which motor proteins can 
migrate, whether the function be to drive actin polymerization in generating protrusive forces at the leading edge of the growth cone or integration of extracellular cues into regulation of microtubule activity (Buck and Zheng, 2002; Dent and Kalil, 2001; Lee et al., 2004; Zhou et al., 2004). Tubulin mutations have been shown to impair neuronal migration into the cortex (Aiken et al., 2017), and can specifically affect UL CPNs (Keays et al., 2007), which rely on a more dynamic, multipolar mode of cytoskeletal reorganization as they extend short processes to “crawl” through established DL CPNs (Miyata et al., 2001; Sakakibara et al., 2014). Mutations in $\alpha$-tubulin specifically have been shown to cause axonal trafficking defects that impair synaptic stability and function without impacting axonal degeneration or neuronal survival (Buscaglia et al., 2020). Potential interactions of Mllt11 with myosins (data not shown) and microtubules (this study) could provide a mechanistic explanation for the impaired migration and morphology seen in Mllt11 mutants. Moreover, our confirmation that Mllt11 associates with $\alpha$-tubulin in fetal brain lysates provides an explanation for the phenocopy of Mllt11 mutants to those affecting tubulin isoforms. The dynamic expression of Mllt11 during UL CPN development also supports its role as a critical regulator of UL migration. Inefficient extension of neurites may also be attributed to impaired trafficking of organelles, ribosomes for local protein synthesis, or vesicles containing secreted cues and growth cone machinery to the axonal periphery (Gonzalez et al., 2016; Kennedy and Ehlers, 2006). Specifically, the potential Mllt11 interactor Myosin 10 we identified by proteomics is known to regulate radial migration of cortical neurons through its affect on the localization of N-cadherin (Lai et al., 2015), and has been implicated in tumor invasion through its role in process extension (Ropars et al., 2016). It is tempting to speculate that altered neuronal trafficking may be why Mllt11 mutant UL neurons showed a progressive loss in the maintenance of an UL projection morphology and the transcription factor programs.

Loss of Mllt11 from the superficial cortex altered levels of UL2/3 specific transcription factors Satb2 and CDP/Cux1, which function in establishment and maintenance of the corpus callosum as well as mature arborescent neuronal morphology and somal packing (Alcamo et al., 2008; Cubelos et al., 2015; He et al., 2021; Rodriguez-Tornos et al., 2016). Importantly, the loss of layer-specific morphology and identity has been demonstrated in postmortem brains from autistic humans (Casanova et al., 2013; Fujimoto et al., 2021; Stoner et al., 2014). These clinical data are reminiscent of the severe dysplastic appearance of superficial CPNs in the cortex of our 
conditional Mllt11 mutants, revealed by the Golgi staining method. It is presently unclear whether the loss of UL-specific gene expression we observed in our Mllt11 mutants, and tubulinopathies more generally, is primarily due to a disordered cytoskeleton, or through some other uncharacterized gene expression pathway functioning downstream of Mllt11. It is important to note that some neuronal subtypes exhibit altered expression of subpopulation-specific markers when cytoskeletal regulation is altered such that connections to target regions cannot be established (Hippenmeyer et al., 2005). This implies that there may be uncharacterized feedback mechanisms between establishment of connectivity and maintenance of expression of CPN subtype-specific transcription factors. Other potential roles for Mllt11 may include binding to $\mathrm{HSPa} 8$ and regulating protein export from the nucleus required for protein degradation (Li et al., 2014). HSPa8 was identified as a potential Mllt11 in our brain lysate proteomics, the meaning of which has yet to be explored. Yet another possible function for Mllt11 may include regulating the Wnt signaling via $\mathrm{T}$ cell factor 7 , which in turn regulates CD44 to promote cell migration and metastasis (Li et al., 2018; Park et al., 2015). As ours is the first study to explore Mllt11 function in the developing brain, additional studies are needed to shed light on whether Mllt11 could be exerting its effect on transcriptional regulation directly,, indirectly via its cytoskeletal-dependent interactions and intracellular trafficking, or both.

In light of this, we showed that the overexpression of Mllt11 promoted migration into the cortical plate, consistent with a cytoskeletal regulatory function. This is also consistent with in vitro studies, which showed that the overexpression of Mllt11 in cancer stem cell lines can promote their proliferation and invasiveness (Tse et al., 2017). Possible downstream effects of Mllt11-dependent cytoskeleton re-organization events may lead to favouring the symmetric divisions of organelles, nuclear components, and polarity protein complexes, all of which favour invasiveness of cells (Nance and Zallen, 2011; Piroli et al., 2019). On the other hand, the transcriptional regulation of Mllt11 by REST has been associated with promoting neuronal differentiation, with high REST expression correlating and low Mllt11 in undifferentiated neuronal tissue, and increasingMllt11 levels associated with decreased REST activity during terminal neuronal differentiation (Hu et al., 2015). This implies that there are potentially two discrete pathways through which Mllt11 regulates neuronal development: a cyctoskeletal regulatory mechanism, and a transcriptional regulatory mechanism. 
Much of what is known about Mllt11 has been discerned from overexpression studies in immortalized cell lines and oncogenic clinical case studies, revealing pathogenic roles in cell process extension, increased invasiveness, and secretion of factors that promote efficient motility of cells (Chang et al., 2008; Li et al., 2006; Lin et al., 2004; Park et al., 2019; Tiberio et al., 2017; Tse et al., 2004). Oncogenesis and neurogenesis share commonalities at the level of cytoskeletal regulation of somatic and nuclear morphology, as well as extension of and trafficking along cytoplasmic processes, allowing for invasion of and migration through tissues. However, it is important to make the distinction between these pathological studies and our current findings on the role of Mllt11. Until the current study, the physiological role for Mllt11 was unclear. In all the published in vitro and oncogenic case studies, Mllt11 is aberrantly overexpressed in nonneuronal tissues either by itself or as a fusion with the chromatin remodelling protein Mll. Despite this, when we examined Mllt11 expression during embryogenesis, we found it to be exclusively localized to developing neurons, and not in another other tissues, such as those deriving from mesoderm or endoderm (Yamada et al., 2014). The expression pattern of $\beta$-gal in the targeted Mllt11 allele confirmed that it is normally exclusively expressed in developing neurons, with the highest levels reflecting UL cortical neurogenesis. Consistent with this, we now show that Mllt11 is required for proper migration, but not neurogenesis of UL CPNs. Furthermore, we also revealed that Mllt11 regulates neurite outgrowth and the maintenance of UL gene expression programs. The loss of Mllt11 led to a reduction of interhemispheric connectivity via reduced crossing of fibres along the corpus callosum. We also characterized a severe dysplasia of CPNs in Mllt11 cKO mutant neonatal brains; a phenotype found in severe neurodevelopmental disorders such as autism. Finally, we provide a possible mechanism of action for Mllt11 to link these phenotypes via its association with microtubules.

In summary, we investigated the role of Mllt11 in development of UL CPNs using a genetic knockout and labeling strategy to target the superficial cortex. Numbers and laminar distribution of UL CPNs were assessed over development, as well CPN neurite morphology. By combining in vitro and in vivo neurite outgrowth and morphology assays, we demonstrate that Mllt11 is required for neuronal invasion in the cortical plate, formation of mature dendritic morphologies characteristic of UL CPNs, and the extension of UL axons across the corpus callosum. Mllt11 interacts with the microtubule cytoskeleton and likely exerts its effect by 
altering cytoskeletal organization during development. Whether this occurs through the stabilization of cytoskeletal architecture or by trafficking of cellular machinery along neuritis will be the subject of future investigations.

\section{MATERIALS AND METHODS}

\section{Animals}

All animal experiments were done according to approved protocols from the IACUC at Dalhousie University. Mice (Mus musculus) carrying a null mutation in the Mllt11 gene were generated using embryonic stem (ES) cell clones obtained from the mouse knockout consortium project (UCDavis KOMP repository, Mllt1 1tmla(KOMP)Mbp). The targeting construct is a "knock-out first, conditional second" approach, which inserts the gene encoding $\beta$ - galactosidase ( $\beta$-gal) exon 2, the protein-coding sequence of the Mllt11 gene. Two independently targeted clones were injected into blastocysts, and the resulting chimeras were mated to BL/6 females to achieve germ-line transmission. Offspring were genotyped by PCR using the following primers: Wild Type F=5'-CGGTCCTGCCTTTGATTCTCAGC-3' and R=5'GCCTACTGCACAAGGTTCTTCTTGG-3' (expected product size: 379bp), Mutant F=5'GAGATGGCGCAACGCAATTAATG-3' and R=5'-AAGCAGTATTTGCTTACTGGCCTGG-3' (expected product size: 274bp). Heterozygotes (Mllt11tmla(KOMP)Mbp/+) were maintained on a C57BL/6 background and crossed with FlpO $^{+-}$(B6.Cg-Tg(Pgkl-flpo)10Sykr/J, 011065, The Jackson Laboratory) mice and converted to a conditional allele via germ line Flp recombinase expression. Resulting Mllt11 $1^{\text {Flox/Flox }}$ offspring were crossed with Ai9 Rosa26TdTomato (B6.CgGt(ROSA)26Sor ${ }^{\text {tm9(CAG-tdTomato }) H z e} / \mathrm{J}, 007909$, The Jackson Laboratory) reporter line to generate Mllt11 $^{\text {Flox/Flox }}$; Rosa26TdTomato ${ }^{+/-}$or Mllt1 $1^{\text {Flox/Flox }}$; Rosa26TdTomato ${ }^{+/+}$offspring. Cux2iresCre mice used in this study to delete Mllt11 in developing UL neurons were previously described (Gil-Sanz et al., 2015; Yamada et al., 2015). Cux2iresCre ${ }^{+/-}$mice $(B 6(C g)$ Cux2<tm1.1(cre)Mull>/Mmmh, Mutant Mouse Resource and Research Center) were crossed with Ai9 (B6.Cg-Gt(ROSA)26Sor ${ }^{\text {tm } 9(\text { CAG-tdTomato }) H z e} / \mathrm{J}, 007909$, The Jackson Laboratory) mice. The resulting Cux2iresCre ${ }^{+/-}$;Rosa26TdTomato ${ }^{+/-}$or Cux2iresCre ${ }^{+/-} ;$Rosa26TdTomato $^{+/+}$offspring were crossed with the Mllt11 $1^{\text {Flox/Flox }}$; Rosa26TdTomato ${ }^{+/+}$line to conditionally knock out Mllt11. Offspring of these crosses were genotyped using the following primers: (Wild Type F=5'CGGTCCTGCCTTTGATTCTCAGC-3' and R=5'-GCCTACTGCACAAGGTTCTTCTTGG-3' 
(expected product size: 379bp), Post Flp/Cre F= F=5'-CGGTCCTGCCTTTGATTCTCAGC-3' and $\mathrm{R}=5$ 'AAGCAGTATTTGCTTACTGGCCTGG-3', and Cre F=5' GTTATAAGCAATCCCCAGAAATG-3' and R=5'-GGCAGTAAAAACTATCCAGCAA3'. We genotyped for the presence of TdTomato using primers $\mathrm{F}=5$ '

TACGGCATGGACGAGCTGTACAAGTAA-3’ and R=5'- CAGGCGAGCAGCCAAGGAAA3 (expected product size: $500 \mathrm{bp}$ ) and allelism was determined with primers $\mathrm{F}=5$ ' -

TCAATGGGCGGGGGTCGTT-3', R=5' -TTCTGGGAGTTCTCTGCTGCC-3', and R=5'CGAGGCGGATCACAAGCAATA-3' (expected product size: 250pb for wild type and $300 \mathrm{bp}$ for mutant)

\section{GST protein expression, Mass Spectrometry, and Immunoprecipitation}

GST-tagged protein expression. MLLT11 was cloned into pGEX-4T-2 vector for GST pulldown assays. BL21 competent cells (Sigma) were transformed with pGEX-4T-2 (GST) and pGEX-4T-2-MLLT11 (GST-MLLT11). Colonies were picked and incubated overnight in $2 \mathrm{~mL}$ $2 \mathrm{xYT}$ media with ampicillin at $37^{\circ} \mathrm{C}$ with shaking. The following day, overnight culture was added to $150 \mathrm{~mL} 2 \mathrm{xYT}$ media with ampicillin and incubated at $37^{\circ} \mathrm{C}$ with shaking until $\mathrm{OD}_{600}$ reached 0.6. Protein expression was induced by adding IPTG (Invitrogen) to the culture to a final concentration of $0.1 \mathrm{mM}$ and shaken at $28^{\circ} \mathrm{C}$ for approx. 4 hours. Cells were pelleted by centrifugation and resuspended in $8 \mathrm{~mL}$ NP-40 lysis buffer $(50 \mathrm{mM}$ Tris $\mathrm{pH} 8.0,150 \mathrm{mM} \mathrm{NaCl}$, $1 \% \mathrm{NP}-40$ ) with protease inhibitor cocktail (Sigma). Cells were nutated at $4{ }^{\circ} \mathrm{C}$ for 10 mins, sonicated on ice (30s on/30s off, for 3 minutes total, level 6 intensity), and again nutated at $4^{\circ} \mathrm{C}$ for 10mins. Lysed cells were pelleted and supernatant collected (protein lysate).

Immobilization of GST or GST-MLLT11 bait protein. GST or GST-MLLT11 proteins were immobilized to Glutathione Sepharose 4B beads (Pierce). GST or GST-MLLT11 protein lysates were added to equilibrated $50 \%$ bead slurry and nutated at $4{ }^{\circ} \mathrm{C}$ for approximately 3 hours. Beads with immobilized protein were collected, washed, and resuspended in PBS to make a 50\% slurry.

GST pull-downs and Mass Spectrometry. Whole embryonic mouse brains were harvested at E15.5 and lysed in NP-40 lysis buffer with protease inhibitor cocktail (Sigma). Whole brain lysates from one embryo were added to either $6 \mu \mathrm{g}$ GST or $6 \mu \mathrm{g}$ GST-MLLT11 bead slurries and nutated overnight at $4{ }^{\circ} \mathrm{C}$. Beads with bound lysate were pelleted, washed, resuspended in sample 
buffer. Samples were heated for $15 \mathrm{mins}$ at $37^{\circ} \mathrm{C}$ and run on an SDS-PAGE gel, then stained with Coomassie Blue (Pierce). Bands were cut from the SDS-PAGE gel and processed by Dalhousie's Biological Mass Spectrometry Core Facility.

Co-immunoprecipitation. HEK293 cells were transfected with myc or myc-MLLT11 vectors using Lipofectamine ${ }^{\mathrm{TM}} 2000$ (Invitrogen). 24 hours later, cells were lysed on ice with Tris- $\mathrm{HCl}$ lysis buffer containing 50mM Tris- $\mathrm{HCl} \mathrm{pH} 7.4,150 \mathrm{mM} \mathrm{NaCl}, 1 \mathrm{mM}$ EDTA, $1 \%$ Triton $\mathrm{X}-100$, and protease inhibitor cocktail (Sigma). Lysed cells were collected, centrifuged, and supernatant used for co-IP. Anti-c-Myc Agarose resin (Pierce) was used, as per manufacturer's protocol, to co-immunoprecipitate myc or myc-MLLT11 and their binding partners. Proteins were eluted from the resin with $50 \mathrm{mM} \mathrm{NaOH}$, neutralized with $1 \mathrm{M}$ Tris $\mathrm{pH} 9.5$, and added to non-reducing sample buffer for Western blot analysis.

\section{Western blots}

E18.5 cortical protein samples were separated on 8\% SDS-PAGE gels for $1 \mathrm{hr}$ at $120 \mathrm{~V}$ and transferred overnight at 20V on to PVDF membranes (BioRad). Blots were probed with rabbit anti-tubulin $\beta 3$ (1:1000, BioLegend), mouse anti-acetylated tubulin (1:20,000, Sigma), mouse anti-GAPDH (1:1000, Invitrogen), and rabbit anti-GAPDH (1:1000, Elabscience). Secondary antibodies were goat anti-rabbit HRP (1:5000, Invitrogen) and goat anti-mouse HRP (1:5000, Invitrogen). Blots were developed with Clarity Western ECL Substrate (BioRad) and imaged on a ChemiDoc ${ }^{\mathrm{TM}}$ Touch Gel Imaging System (BioRad). Band densitometry was done using Image Lab Software (BioRad).

\section{qPCR}

To confirm the loss of Mllt11 RNA, E18.5 RNA was extracted from cortices of 3 genotypic conditional knockouts and 4 controls using the RNeasy Micro Kit (Qiagen). RNA was reverse transcribed to cDNA using the SuperScript ${ }^{\mathrm{TM}}$ II Reverse Transcriptase Kit (Invitrogen). qPCR reactions were carried out using the SensiFAST ${ }^{\mathrm{TM}}$ SYBR ${ }^{\circ}$ No-ROX Kit (Bioline) with the following primers for Mllt11 and internal control GAPDH: Mllt11 F=5'-

GAACTGGATCTGTCGGAGCT-3 and R=5'-GCGCTCTCCAGAAGTTGAAG-3', GAPDH $\mathrm{F}=5$ ' - ACCACAGTCCATGCCATCAC-3' and R=5' -TCCACCACCCTGTTGCTGTA-3'. The source for GAPDH primers was (Weng et al., 2014). 


\section{Histology}

Fetal brains were dissected out and fixed in $4 \%$ paraformaldehyde (PFA) for 4-8 hours, depending on embryonic stage, before being equilibrated in sucrose, embedded in Optimum Cutting Temperature (OCT) compound (Tissue-Tek, Torrance, CA), and cryosectioned at $12 \mu \mathrm{m}$. Cortex morphology was assessed by DAPI staining. $\beta$-Gal staining was performed on cryosectioned slides using the $\beta$-Gal Tissue Stain Kit (Millipore). Immunohistochemistry was conducted on E14.5-E18.5 brains as described previously (Iulianella et al., 2008).

Immunohistochemistry was conducted using the following antibodies: rabbit anti-CDP/Cux1 (1:100; Santa Cruz), mouse anti-Satb2 (1:250; Abcam), rat anti-Bcl11b/Ctip2 (1:500; Abcam), rabbit anti-Tbr1 (1:200; Abcam), goat anti-Nestin (1:250; Santa Cruz), rabbit anti-Cleaved Caspase 3 (CC3, 1:500; Cell Signaling Technology), chicken anti-Tbr2 (1:500; Millipore), rabbit anti-Pax6 (1:500; Abcam), goat anti-Sox2 (1:200; Santa Cruz), mouse anti-NeuN (1:200; Millipore), rat anti-Tbr2 (1:200; eBioscience), mouse anti-Ki67 (1:25; BD Biosciences) and mouse anti-neurofilament 2H3 (1:200; DSHB, University of Iowa). Species-specific AlexaFluor 488-, 568-, 594-, and/or 647-conjugated IgG (1:1500; Invitrogen) secondary antibodies were used to detect primary antibodies.

In situ hybridization was performed on 30mm frozen sections obtained from E18.5, P7, P14, P21, and P28 Control and cKO (N=3/genotype) brains fixed overnight as previously described (Yamada et al., 2014) using an Mllt11 riboprobe (Chen et al., 2008; Toma et al., 2014). For EdU birth dating studies, dams were injected intraperitoneally with $30 \mathrm{mg} / \mathrm{kg}$ body weight of EdU (Invitrogen) at E14.5 and E16.5 and E18.5 and sacrificed at E14.5 or E18.5. Sections were immunostained using the Click-It Kit according to the manufacturer's protocol (Invitrogen). For Golgi stains, brains were harvested from mice at P28 and subjected to the FD Rapid GolgiStain Kit (FD Neurotechnologies, Inc.) as per manufacturer instructions. Crude sections were cut and mounted on slides with Permount Mounting Medium (Fisher Scientific). 4 brains were analyzed per genotype and 30 neurons per individual were analyzed.

Microscopy. Images were captured using a Zeiss AxioObserver fluorescence microscope equipped with an Apotome 2 structured illumination device, 10x, 20x, and a Hamamatsu Orca Flash v4.0 digital camera. $\beta$-Gal and Mllt11 in situ staining was captured using an upright Zeiss PrimoStar compound microscope with an ERc5s colour camera. Images were processed using Zen software (Zeiss, Germany) and Photoshop CS6 (Adobe, San Jose, CA). 


\section{Primary cortical cell culture and immunocytochemistry}

Cortices were microdissected from E18.5 embryos, digested in trypsin (Pierce), manually triturated and plated on $35 \mathrm{~mm}$ well onto poly-D-lysine coated coverslips at a density of 150,000 cells for the neurite outgrowth assay or 500,000 for cellular localization experiments. Cells were plated in medium containing DMEM with $10 \%$ FBS and 1\% penicillin/streptomycin and 4 hours after plating, media was completely removed and replaced with Neurobasal media containing B27+ (Gibco/Invitrogen), 1\% penicillin/streptomycin, and L-glutamine. Cells were cultured for 24 hours or 1 week in a $37^{\circ} \mathrm{C}$ incubator containing $5 \% \mathrm{CO} 2$, then fixed for 10 minutes in $4 \%$ paraformaldehyde. Immunocytochemistry was conducted using the following antibodies: mouse anti-Tau (1:200; Abcam), rabbit anti-MAP2 (1:1000; Abcam), rabbit anti-Mllt11 (1:300, Abcam), rabbit anti-Tuj1 (1:1000, Biolegend), and mouse anti-acetylated $\alpha$-tubulin (1:1000, Millipore Sigma).

\section{In utero electroporation and cDNA constructs}

In utero electroporation was conducted using standard methodology under sterile surgical conditions (Saito, 2006). Endotoxin-free DNA was prepared according to the instructions of the manufacturer (Qiagen) and injected at $1.5 \mu \mathrm{g} / \mu \mathrm{l}$ into the telencephalic vesicles of embryos in time-staged pregnant females anesthetized under inhalable isoflurane $(5 \mathrm{~L} / \mathrm{min})$. A small incision was made on the ventral midline of anesthetized pregnant FVB females under a sterile field treatment. Single uteri containing the E13.5 fetuses were extruded and electric current was delivered across the fetal brains as 5 pulses for 50ms at $900 \mathrm{~ms}$ intervals using tweezer-style electrodes linked to the pulse generator CUY21 Vivo SQ (Sonidel). The embryos were returned in the body cavity, the peritoneum was sutured and the skin was stapled. Experimental plasmids used were Mllt11-ires-eGFP, and control plasmids included pIRES2-EGFP (Clonetech) or pCIG (Addgene). To ensure comparable development staging, for each dam one uterus was electroporated with the experimental construct and the other with the control vector. Fetuses were allowed to survive for 2 days until E15.5 after which they were processed for cryosectioning to evaluate GFP expression and cortical layer development by immunostaining. N=3 Mllt11-eGFP and $\mathrm{N}=4 \mathrm{eGFP}$ control fetal brains were analysed. Line graphs and statistical testing were conducted using Graphpad Prism V5.0d software, with results shown as mean \pm SD. Statistical differences were determined with a Welch's t-test. 


\section{DiI tracing of callosal projections}

Brains of E18.5 embryos were removed and embedded in $7 \%$ low gelling temperature agarose in DMEM medium with $1 \%$ penicillin/streptomycin. Embedded brains were crudely sectioned by hand at a thickness of approximately $1-2 \mathrm{~mm}$. Approximately $0.5 \mu \mathrm{L}$ of DiI (Invitrogen) was injected into the cortical white matter tracts at rostral and caudal axial levels and incubated for 8 hours in Tyrode's solution. Tissues were then fixed overnight at $4^{\circ} \mathrm{C}$ in $4 \%$ paraformaldehyde before incubating for 2 weeks at room temperature in PBS with 1\% penicillin/streptomycin. Images were captured using Zeiss V16 Axiozoom stereomicrscope.

\section{Image sampling, quantification, statistics, and Sholl analysis}

For analysis of immunostaining markers and EdU, counting frames $(100 \mu \mathrm{m} \times 100 \mu \mathrm{m})$ were placed in a vertical strip along the somatosensory (S1) cortex with the first counting frame along the edge of the ventricle. At least 3 histological sections within the somatosensory cortex from 3-8 different animals were analysed for each immunostain, EdU or electroporated vector. Cells that were positively labelled for both DAPI and the marker were counted within each frame using ImageJ (FIJI) (Schindelin et al., 2012). Analysis of directionality and amount of radial glia stain in the E18.5 neocortex using Nestin stained images, captured with identical acquisition parameters, was assessed using features found in FIJI (Image J). The 'directionality' plugin was used to calculate the percentage of fibres in the image aligned in the same direction. Automatic thresholds were then applied and the area covered by Nestin staining was measured and expressed as a percentage of the total image area. From each of these measurements population averages of WT and mutant were compared. For analysis of primary cortical cell culture, neurites were traced and measured and Sholl analyses were conducted using the "simple neurite tracer" plugin (FIJI). At least 50 cells were counted per individual $(n=3)$. Image montages were assembled using Photoshop CS6.

To ensure consistency among samples, cell counts were restricted to the presumptive S1 (somatosensory cortex) of the embryonic brain. In all experiments, 3-6 Mllt11 KO and control embryos were used for quantification analysis using the unbiased and systematic sampling method we described previously (Yamada et al., 2015). Counts for Ctip2, Tbr1, Cux1, Tbr2, NeuN, Sox2, Pax6, Ki67, CC3, and Satb2 are represented as line graphs, which represent the proportion of DAPI stained cells expressing those markers. For EdU, TdTomato, and DAPI 
distribution analyses, proportion of total stained cells expressing markers within each cortical bin was quantified to ensure counting methods were consistent with previous studies (Chen et al., 2008; Lawrenson et al., 2017; Reyes et al., 2019). For the electroporation experiments, 3-5 independent samples were analysed. Bar charts, line graphs, and statistical testing were conducted using Graphpad Prism V5.0d software, with results shown as mean \pm SD. In all quantification studies, statistical differences were determined with Welch's t-test (two tailed), or paired t-test in the case of Sholl analyses, with significance level set at $\mathrm{P} \leq 0.05$ (*P $\leq 0.05$, $* * \mathrm{P} \leq 0.01, * * * \mathrm{P} \leq 0.001, * * * * \mathrm{P} \leq 0.0001)$.

\section{ACKNOWLEDGMENTS}

A.I. lab gratefully acknowledges funding support for this project from the Canadian Institutes of Health Research (CIHR PJT-388914). We thank Sarah Whitehead, Emily Capaldo, Jessica Clark, and Di Shao for maintenance of mouse transgenic colonies, and Marina Gertsenstein at the Centre for Phenogenomics in Toronto, Canada, for assistance in generating chimeric mice. We thank Leanne Clattenburg for advice on GST pull down experiment.

\section{FIGURE LEGENDS}

\section{Figure 1: Mllt11 loss perturbed formation of UL CPNs without affecting deeper layers.}

(A-E) Coronal cortical slices at E18.5 stained for cortical layer markers with IHC. (A) Mltt11 conditional knockout (cKO) cortical slices stained for Satb2 displayed a severe decrease in expression at E18.5 compared to controls (ctrl). (B) CDP/Cux1 levels were decreased in Mllt11 cKOs compared to controls at E18.5. (C) Distribution of TdTomato expression in the cortex at E18.5 was comparable between controls and cKOs. (D-E) Tbr1 (D) and Ctip2 (E) levels were comparable between controls and cKOs but their expression domains exhibited a downward shift at E18.5. Line charts represent percentage of positive cells normalized to DAPI+ nuclei per $100 \mu \mathrm{m} \times 100 \mu \mathrm{m}$ bin for (A-B) and (D-E) and percentage of total TdTomato+ cells per $100 \mu \mathrm{m} \times$ $100 \mu \mathrm{m}$ bin for (C). Welch's t-test, (A, D, E) N=4, (B-C) N=5. Data presented as mean \pm SD. n.s., not significant. $* \mathrm{P} \leq 0.05 ; * * \mathrm{P} \leq 0.01 ; * * * \mathrm{P} \leq 0.001 ; * * * * \mathrm{P} \leq 0.0001$. Scale bar: $50 \mu \mathrm{m}$ for $(\mathrm{A}-\mathrm{O})$. VZ, ventricular zone. 
Figure 2: EdU birthdating demonstrating a CPN migratory defect in Mllt11 cKO mutants. (A-B) EdU birthdating of UL CPNs by injection at E14 showed altered distribution in cKOs at E18.5, reflecting a downward shift in expression correlating with the decreased cortical thickness. (B) Moderate downward shift in migrating CPNs in cKOs relative to controls following an EdU pulse at E16 (B). (C) Proliferating cells labeled with a short pulse of EdU at E14 showed no significant differences in numbers or distribution between controls and cKOs. (D) No significant difference in numbers of nuclei labeled by a short pulse of EdU at E18 populating most bins in cKOs vs. controls, but a greater proportion of cells were retained in the $\mathrm{cKO} \mathrm{VZ}$ region. Line charts represent percentage of positive cells per $100 \mu \mathrm{m} \times 100 \mu \mathrm{m}$ bin as a proportion of total EdU+ cells. Welch's t-test, $(A, C) N=4,(B, D) N=3$. Data presented as mean \pm SD. n.s, not significant. $* \mathrm{P} \leq 0.05 ; * * \mathrm{P} \leq 0.01 ; * * * \mathrm{P} \leq 0.001 ; * * * * \mathrm{P} \leq 0.0001$. Scale bar: $50 \mu \mathrm{m}$ for $(\mathrm{A}-\mathrm{O})$. VZ, ventricular zone.

\section{Figure 3: Mllt11 overexpression promoted migration into the cortical plate.}

(A-B) E15.5 coronal cortical sections after electroporation at E13.5 wither either a control eGFP (left) or Mllt11-ires-eGFP (Mllt11-eGFP) bicistronic plasmid (right). (B) Mllt11-eGFP electroporation promoted migration into the CP, identified by Tbr1 staining. (C) Control GFP (eGFP-control) electroporated cells remained mostly within the SVZ/VZ, identified by Tbr2 staining. (D-E) Localization of eGFP-control and Mllt11-eGFP+ cells (D) quantified by cortical region (E). Welch's t-test; N=3 Mllt11-eGFP, N=4 eGFP controls. Data presented as mean \pm SD. $* \mathrm{P} \leq 0.05$. Scale bar: $25 \mu \mathrm{m}$ for (A). CP, cortical plate; IZ intermediate zone; SVZ, subventricular zone; VZ, ventricle zone.

\section{Figure 4: Formation of white matter tracts and callosal projections is impaired in the}

\section{Mllt11 cKO cortex.}

(A) Image of cortical white matter tracts labeled with neurofilament at E16.5. Brackets indicated decreased white matter. (B-C) Quantification of cortical white matter thickness (B) and white matter thickness as a proportion of total cortical thickness (C) showed significant decrease in white matter in cKOs relative to controls at E16.5. (D) Image of cortical white matter tracts labeled with neurofilament at E18.5. Brackets show decreased white matter. (E-F) Quantification of cortical white matter thickness (E) and white matter thickness as a proportion of total cortical 
thickness (F) displayed a decrease in cKOs at E18.5. (G-H) Coronal sections of E18.5 cortices at rostral (upper panels) and caudal (lower panels) axial levels labeled with neurofilament (G) or traced with DiI (H). (G) Neurofilament labeling of the corpus callosum was significantly decreased in cKO compared to controls but labeling of the internal capsule was unaffected. $(\mathrm{H})$ DiI labeling was absent in the corpus callosum of cKO slices while control cortices displayed crossing fibres labeled by DiI. Rostrally, the internal capsule was traced comparably in control and cKO cortices. Welch's t-test, (A-C) $\mathrm{N}=5,(\mathrm{D}-\mathrm{F}) \mathrm{N}=4,(\mathrm{H}) \mathrm{N}=3$. Data presented as mean \pm SD. $* * * * \mathrm{P} \leq 0.0001$. Scale bar: $200 \mu \mathrm{m}$ for $(\mathrm{A}) ; 50 \mu \mathrm{m}$ for $(\mathrm{B})$. ctx, cortex; cc, corpus callosum; str, striatum; th, thalamus; ic, internal capsule; VZ, ventricular zone; WM, white matter.

\section{Figure 5: Mllt11 associates and colocalizes with acetylated $\alpha$-tubulin in growing neurites.} (A) Co-immunoprecipitation of acetylated $\alpha$-tubulin (Actub) with Myc-tagged Mllt11 in whole cell lysates of HEK293 cells compared to a myc programmed control. (B) Co-IP probed with Mllt11 antibody showing IP signal restricted to lanes containing M1lt11. (C-D) Western blots of E18.5 brain lysates probed for Tubb3 (C) and acetylated $\alpha$-tubulin (D) show no differences in levels between controls and cKOs. (E) Bar graph of the ratio of acetylated tubulin to Tubb3 normalized to GAPDH. Conditional mutants trend toward an increased ratio (not significant). (FG) Primary cortical neurons cultured for 24 hours (F) or 7 days (G) post-plating. (F) After 24 hours, Mllt11 colocalizes with Tubb3 in the growth cone and at swellings along the distal axon as indicated by arrowheads (f'), and in the soma (F). After 7 days, Mllt11 was primarily found along proximal portions of the axon where it colocalizes with acetylated $\alpha$-tubulin $(\mathrm{G})$. Welch's t-test, $\mathrm{N}=4$ (controls and cKOs). Data presented as mean \pm SD. n.s, not significant (i.e. P>0.05). Scale bar: $20 \mu \mathrm{m}$.

\section{Figure 6: Mllt11 loss decreased neurite outgrowth and branching complexity in vitro.}

(A) Primary cortical neurons derived form E18.5 brains cultured for 24 hours post-plating. Neurites identified with MAP2 staining (red). (B-C) 24 hours post-plating, primary neurite length (B) and total neurite length (C) were significantly decreased in cKO neurons relative to controls (D). The proportion of total primary neurite length was significantly increased in cKO neurons relative to controls. (E-G) Quantification of decreases in total neurite length (E) and branching complexity $(\mathrm{F})$ of cKO relative to control primary cortical neurons cultured for 7 days post- 
plating (G). Paired t-test, for both controls and cKOs $\mathrm{N}=150$ neurons (50 neurons/individual X 3 individuals). Data presented as mean $\pm \mathrm{SD}$. $* \mathrm{P} \leq 0.05 ; * * \mathrm{P} \leq 0.01 ; * * * \mathrm{P} \leq 0.001, * * * * \mathrm{P} \leq 0.0001$. Scale bar: $20 \mu \mathrm{m}$ for (A); $50 \mu \mathrm{m}$ for (E). HPP, hours post-plating; DPP, days post-plating.

Figure 7: Decreased neurite outgrowth and branching complexity in UL CPNs of the Mllt11 cKO cortex.

(A-C) Corresponding images from coronal sections of P28 Golgi stained control and cKO cortices. (A) 10x magnification view of overall cortical morphology. cKOs displayed dark, densely stained punctate structures in the upper cortical layers, which were absent in controls. (BC) 200x magnification view of sample UL2/3 CPNs varying in morphology between control and cKO neurons. (D-E) Quantification of neurite length (D) and branching complexity (E) within UL2/3 showed reduced neurite length and branching complexity in cKOs compared to controls. Length measurements analyzed with Welch's t-test, layer-specific Sholl analysis compared with paired t-test, $\mathrm{N}=4$ (controls and cKOs), 30 neurons/individual. Data presented as mean $\pm \mathrm{SD}$. $* \mathrm{P} \leq 0.05 ; * * \mathrm{P} \leq 0.01$. Scale bar: $100 \mu \mathrm{m}$ for $(\mathrm{A}) ; 50 \mu \mathrm{m}$ for $(\mathrm{B}-\mathrm{C})$.

\section{SUPPLEMENTARY FIGURE LEGENDS}

\section{Figure S1: Mllt11 targeting strategy and expression timeline.}

(A) Graphic representation of the targeting construct inserted into the Mllt11 locus. Mllt11 expression can be evaluated by $\beta$-Gal staining due to the insertion of lac Z cDNA in the targeted allele. A conditional knockout allele can be garneted by the removal of the lac $Z$ and selection cassette by germline Flp recombination. (B) A reference coronal section of a control brain with a boxed region to indicate the area sampled in panel (C) Mllt11 expression in the cortex and hippocampus from P7 to P28. RNA levels declined in the cortex and were indistinguishable from background by P28. Expression in the hippocampus remained detectable throughout all stages evaluated. Scale bar: $100 \mu \mathrm{m}$.

\section{Figure S2: Mllt11 conditional knockout validation by qPCR and ISH.}

(A) Quantitation (q)PCR fluorescence levels of control and cKO cortices normalized to internal control GAPDH. (B) Fold change of Mllt11 cDNA transcript levels was significantly decreased in cKO relative to control brains. (C-D) Images of ISH of Mllt11 riboprobe on P7 control and 
cKO cortices (C) and hippocampi (D) showed decreased labeling in the superficial cortex, corresponding to the $\mathrm{Cux} 2$-expressing region. $\mathrm{CP}$, cortical plate; $\mathrm{RFU}$, relative fluorescence units. Welch's t-test, (A-B) $\mathrm{N}=4$ controls, 3 cKOs, (C-D) $\mathrm{N}=3$. Data presented as mean $\pm \mathrm{SD}$. $* * \mathrm{P} \leq 0.01$. Scale bar: $100 \mu \mathrm{m}$.

\section{Figure S3: Cux2iresCre-driven TdTomato expression in the pallial cortex and corpus callosum.}

(A-B) Cux2iresCre driven TdTomato reporter expression was highest in UL2/3 of the cortex (A) and the corpus callosum (B) at P7, corresponding to regions where the excision event occurs. Scale bar: $100 \mu \mathrm{m}$.

Figure S4: Mllt11 is expressed in the developing cortical plate and Mllt11 loss affects the laminar distribution of cells.

(A) Coronal sections of the targeted Mllt11 locus (which inserted a lacZ cDNA) showing Mllt11 expression across four time points during cortical neurogenesis through $\beta$-Gal staining. At E14.5, $\beta$-Gal expression was most intense in the cortical plate $(\mathrm{CP})$, corresponding to UL neurogenesis. By E16.5 $\beta$-Gal staining intensity shifted to the superficial cortex where UL CPNs were accumulating. (B-D) Body weight (B), brain weight (C), and brain weight as a percentage of body weight (D) showed no difference between control and conditional knockouts (cKOs) at E18.5. (E) cKO cortices were thinner than controls at E18.5. (F-H) Thinning of the cKO cortex was progressive, with thicknesses being comparable at E14.5 (F) but exhibited reduced thickness and reduced distribution of cells in the superficial cortex at E16.5 (G), which increased in severity by E18.5 (H). Total cell counts are shown as bar graphs. Line charts represent percentage of positive cells normalized to DAPI+ nuclei per $100 \mu \mathrm{m}$ x $100 \mu \mathrm{m}$ bin. Welch's t-test, (B-D) $\mathrm{N}=8,(\mathrm{~F}, \mathrm{H}) \mathrm{N}=5,(\mathrm{G}) \mathrm{N}=6$. Data presented as mean \pm SD. n.s, not significant. $* \mathrm{P} \leq 0.05$;

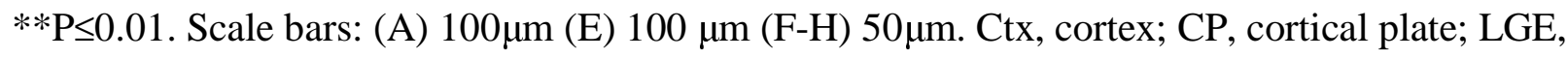
lateral ganglionic eminence; MGE, medial ganglionic eminence; MZ, marginal zone; PP, preplate; SP, subplate; VZ, ventricular zone. 


\section{Figure S5: Loss of Mllt11 had no impact on programmed cell death.}

(A-B) Cortical slices at E14.5 (A) and E18.5 (B) showed no differences in the levels of cleaved caspase-3 (CC3) in cKOs relative to controls. (C) CC3 staining in the retrosplenial area at comparable levels, which normally has enhanced apoptosis, included as a control for CC3 staining. White arrowheads indicate positive labeling. $\mathrm{N}=3$ controls, 5 cKOs. Scale bar: $50 \mu \mathrm{m}$. VZ, ventricular zone.

Figure S6: Neural progenitor populations were largely unaffected in Mllt11 cKO mutants. (A-C) Coronal cortical slices stained for Pax6 with IHC was unaltered in cKO cortices relative to controls at E14.5 (A) E16.5 (B) and E18.5. (D-F) Sox2 levels were largely similar between cKO and controls at E14.5 (D) E16.5 (E) and E18.5 (F). (G-I) Tbr2 expression was also largely unaltered in cKOs at E14.5 $(\mathrm{G})$ and E16.5 $(\mathrm{H})$, but showed a significant trend towards decreased levels normalized to DAPI+ nuclei immediately above the Tbr2+ progenitor domain at E18.5 (I). Line charts represent percentage of positive cells normalized to DAPI+ nuclei per $100 \mu \mathrm{m} x$ $100 \mu \mathrm{m}$ bin. Welch's t-test, (A-C, F-I) N=4, (D-E) N=3. Data presented as mean \pm SD. n.s, not significant. ${ }^{*} \mathrm{P} \leq 0.05 ; * * \mathrm{P} \leq 0.01 ; * * * \mathrm{P} \leq 0.001$. Scale bar: $50 \mu \mathrm{m}$ for $(\mathrm{A}-\mathrm{I})$. VZ, ventricular zone.

\section{Figure S7: Progressive loss of UL CPN marker expression in Mllt11 cKO mutants.}

(A-B) Coronal slices stained for Satb2 with IHC were similar between Mllt11 cKO and controls at E14.5 (A) but displayed a tendency toward decreased numbers in upper bins and a downward shift at E16.5 (B). (C-D) CDP/Cux1 levels were largely normal at E14.5 (C) but began to decrease at E16.5 (D). (E-F) TdTomato levels were comparable between control and cKO at E14.5 (E) and E16.5 (F). (G-H) Tbr1 levels and localization were comparable between control and cKO at E14.5 (G) and E16.5 (H). (I-J) Ctip2 expression and localization were largely unaltered in cKO at E14.5 (I) and E16.5 (J). Line charts represent percentage of positive cells normalized to DAPI+ nuclei per $100 \mu \mathrm{m} \times 100 \mu \mathrm{m}$ bin for (A-D) and (G-J) and percentage of total TdTomato+ cells per $100 \mu \mathrm{m} \times 100 \mu \mathrm{m}$ bin for $(\mathrm{E}-\mathrm{F})$. Welch's t-test, (A-D, G, I-J) N=4, (E) N=5 controls, $\mathrm{N}=6 \mathrm{cKOs},(\mathrm{H}) \mathrm{N}=4$ controls, $\mathrm{N}=5$ cKOs. Data presented as mean $\pm \mathrm{SD}$. n.s, not significant. $* \mathrm{P} \leq 0.05$. Scale bar: $50 \mu \mathrm{m}$ for $(\mathrm{A}-\mathrm{J})$. VZ, ventricular zone. 
Figure S8: CPNs lacking Mllt11 maintained neuronal identity.

(A-B) Coronal cortical slices at E16.5 (A) and E18.5 (B) showing expression of NeuN was maintained though the cortical plate, but was compressed and shifted downward in cKOs relative to controls. (C) GFAP staining in the cortex showed comparable levels between cKOs and controls. Unaltered NeuN and GFAP levels suggested glial and neuronal fates are maintained in controls and cKOs. Welch's t-test, $\mathrm{N}=3$. Data presented as mean $\pm \mathrm{SD}$. $* \mathrm{P} \leq 0.05 ; * * \mathrm{P} \leq 0.01$; $* * * \mathrm{P} \leq 0.001, * * * * \mathrm{P} \leq 0.0001$. Scale bar: $50 \mu \mathrm{m}$. VZ, ventricular zone.

Figure S9: CPNs lacking Mllt11 failed to fully invade cortical plate but do not acquire a DL identity.

(A-B) Coronal cortical slices of control and cKO and close-up views (a',b') of mice injected with EdU at E14.5 (A-a') or E16.5 (B-b') and harvested at E18.5. Slices are co-labeled with DL CPN marker Ctip2 (A-B). Significantly less EdU+ cells invaded the cortical plate of cKO relative to control cortices. Welch's t-test, $\mathrm{N}=3$ (controls and cKOs). Data presented as mean \pm SD. $* \mathrm{P} \leq 0.05$. Scale bar: $50 \mu \mathrm{m}$ for $(\mathrm{A}-\mathrm{D})$. VZ, ventricular zone.

Figure S10: Radial glial fibres were unaltered in Mllt11 cKOs.

(A) Coronal cortical slice at E14.5 showing expression of Nestin in controls vs. Mllt11 cKOs. (BD) Representative angle (B), area (C) and dispersion (D) showed no significant differences between controls and cKO. (D) Nestin expression in the cortex at E16.5. (E-G) Representative angle $(\mathrm{E})$, area $(\mathrm{F})$ and dispersion $(\mathrm{G})$ showed no significant differences between control and cKO cortices at E16.5. (I) Cortical expression of Nestin at E18.5. (J-L) Representative angle (J), area $(\mathrm{K})$ and dispersion $(\mathrm{L})$ show no significant differences between control and $\mathrm{cKO}$ at E18.5, but dispersion (L) trended towards an increase in cKOs relative to controls. Welch's t-test, (A-D) $\mathrm{N}=4$, (E-H) $\mathrm{N}=5$, (I-L) N=3. Data presented as mean \pm SD. n.s, not significant. Scale bar: $50 \mu \mathrm{m}$ for (A, E, I). VZ, ventricular zone.

Figure S11: Cajal-Retzius cells in Cortical Layer 1 are unaffected by Mllt11 loss. (A-B) Pial sections at E14.5 (A) and E18.5 (B) showed comparable levels of expression of CR markers P73 and Reelin in Layer 1 of cKOs vs. controls. Total co-labeled cells are shown as bar graphs. Welch's t-test, $N=3$. Data presented as mean \pm SD. n.s, not significant. Scale bar: $50 \mu m$. 


\section{REFERENCES}

Aboitiz, F. and Montiel, J. (2003). One hundred million years of interhemispheric communication: the history of the corpus callosum. Braz J Med Biol Res 36, 409-420.

Aiken, J., Buscaglia, G., Bates, E. A. and Moore, J. K. (2017). The alpha-Tubulin gene TUBA1 A in Brain Development: A Key Ingredient in the Neuronal Isotype Blend. $J$ Dev Biol 5(3):8 doi: 10.3390/jdb5030008.

Alcamo, E. A., Chirivella, L., Dautzenberg, M., Dobreva, G., Farinas, I., Grosschedl, R. and McConnell, S. K. (2008). Satb2 regulates callosal projection neuron identity in the developing cerebral cortex. Neuron 57, 364-377.

Arlotta, P., Molyneaux, B. J., Chen, J., Inoue, J., Kominami, R. and Macklis, J. D. (2005). Neuronal subtype-specific genes that control corticospinal motor neuron development in vivo. Neuron 45, 207-221.

Ayala, R., Shu, T. Z. and Tsai, L. H. (2007). Trekking across the brain: The journey of neuronal migration. Cell 128, 29-43.

Bahi-Buisson, N., Poirier, K., Boddaert, N., Saillour, Y., Castelnau, L., Philip, N., Buyse, G., Villard, L., Joriot, S., Marret, S., et al. (2008). Refinement of cortical dysgeneses spectrum associated with TUBA1A mutations. J Med Genet 45, 647-653.

Bahi-Buisson, N., Poirier, K., Fourniol, F., Saillour, Y., Valence, S., Lebrun, N., Hully, M., Bianco, C. F., Boddaert, N., Elie, C., et al. (2014). The wide spectrum of tubulinopathies: what are the key features for the diagnosis? Brain 137, 1676-1700.

Bellion, A., Baudoin, J. P., Alvarez, C., Bornens, M. and Metin, C. (2005). Nucleokinesis in tangentially migrating neurons comprises two alternating phases: Forward migration of the Golgi/centrosome associated with centrosome splitting and myosin contraction at the rear. J Neurosci 25, 5691-5699.

Bignami, A. and Dahl, D. (1977). Specificity of Glial Fibrillary Acidic Protein for Astroglia. $J$ Histochem Cytochem 25, 466-469.

Breuss, M., Heng, J. I. T., Poirier, K., Tian, G. L., Jaglin, X. H., Qu, Z. D., Braun, A., Gstrein, T., Ngo, L., Haas, M., et al. (2012). Mutations in the beta-Tubulin Gene TUBB5 Cause Microcephaly with Structural Brain Abnormalities. Cell Rep 2, 1554-1562.

Breuss, M. W., Nguyen, T., Srivatsan, A., Leca, I., Tian, G., Fritz, T., Hansen, A. H., Musaev, D., McEvoy-Venneri, J., James, K. N., et al. (2017). Uner Tan syndrome caused by a homozygous TUBB2B mutation affecting microtubule stability. Hum Mol Genet 26, 258-269.

Britanova, O., de Juan Romero, C., Cheung, A., Kwan, K. Y., Schwark, M., Gyorgy, A., Vogel, T., Akopov, S., Mitkovski, M., Agoston, D., et al. (2008). Satb2 is a postmitotic determinant for upper-layer neuron specification in the neocortex. Neuron 57, 378-392.

Brock, S., Stouffs, K., Scalais, E., D'Hooghe, M., Keymolen, K., Guerrini, R., Dobyns, W. B., Di Donato, N. and Jansen, A. C. (2018). Tubulinopathies continued: refining the phenotypic spectrum associated with variants in TUBG1. Eur J Hum Genet 26, 11321142.

Buck, K. B. and Zheng, J. Q. (2002). Growth cone turning induced by direct local modification of microtubule dynamics. J Neurosci 22, 9358-9367.

Buscaglia, G., Northington, K. R., Moore, J. K. and Bates, E. A. (2020). Reduced TUBA1A Tubulin Causes Defects in Trafficking and Impaired Adult Motor Behavior. Eneuro 7(2): ENEURO.0045-20.2020 doi: 10.1523/ENEURO.0045-20.2020. 
Campbell, K. and Gotz, M. (2002). Radial glia: multi-purpose cells for vertebrate brain development. Trends Neurosci 25, 235-238.

Casanova, M. F., El-Baz, A. S., Kamat, S. S., Dombroski, B. A., Khalifa, F., Elnakib, A., Soliman, A., Allison-McNutt, A. and Switala, A. E. (2013). Focal cortical dysplasias in autism spectrum disorders. Acta Neuropathol Com 1:67 doi: 10.1186/2051-5960-1-67.

Chai, X. J., Forster, E., Zhao, S. T., Bock, H. H. and Frotscher, M. (2009). Reelin Stabilizes the Actin Cytoskeleton of Neuronal Processes by Inducing n-Cofilin Phosphorylation at Serine3. J Neurosci 29, 288-299.

Chai, X. J., Zhao, S. T., Fan, L., Zhang, W., Lu, X., Shao, H., Wang, S. B., Song, L. Z., Failla, A. V., Zobiak, B., et al. (2016). Reelin and cofilin cooperate during the migration of cortical neurons: a quantitative morphological analysis. Development 143, 1029-1040.

Chang, X. Z., Li, D. Q., Hou, Y. F., Wu, J., Lu, J. S., Di, G. H., Jin, W., Ou, Z. L., Shen, Z. Z. and Shao, Z. M. (2008). Identification of the functional role of AF1Q in the progression of breast cancer. Breast Cancer Res $\operatorname{Tr}$ 111, 65-78.

Chen, B., Wang, S. S., Hattox, A. M., Rayburn, H., Nelson, S. B. and McConnell, S. K. (2008). The Fezf2-Ctip2 genetic pathway regulates the fate choice of subcortical projection neurons in the developing cerebral cortex. P Natl Acad Sci USA 105, 1138211387.

Clark, D., Dedova, I., Cordwell, S. and Matsumoto, I. (2007). Altered proteins of the anterior cingulate cortex white matter proteome in schizophrenia. Proteom Clin Appl 1, 157-166.

Cubelos, B., Briz, C. G., Esteban-Ortega, G. M. and Nieto, M. (2015). Cux1 and Cux2 Selectively Target Basal and Apical Dendritic Compartments of Layer II-III Cortical Neurons. Dev Neurobiol 75, 163-172.

Cubelos, B., Sebastian-Serrano, A., Kim, S., Moreno-Ortiz, C., Redondo, J. M., Walsh, C. A. and Nieto, M. (2008a). Cux-2 controls the proliferation of neuronal intermediate precursors of the cortical subventricular zone. Cereb Cortex 18, 1758-1770.

Cubelos, B., Sebastian-Serrano, A., Kim, S. H., Redondo, J. M., Walsh, C. and Nieto, M. (2008b). Cux-1 and Cux-2 control the development of Reelin expressing cortical interneurons. Dev Neurobiol 68, 917-925.

Cushion, T. D., Paciorkowski, A. R., Pilz, D. T., Mullins, J. G. L., Seltzer, L. E., Marion, R. W., Tuttle, E., Ghoneim, D., Christian, S. L., Chung, S. K., et al. (2014). De Novo Mutations in the Beta-Tubulin Gene TUBB2A Cause Simplified Gyral Patterning and Infantile-Onset Epilepsy. Am J Hum Genet 94, 634-641.

D'Amour, K. A. and Gage, F. H. (2003). Genetic and functional differences between multipotent neural and pluripotent embryonic stem cells. P Natl Acad Sci USA 100, 11866-11872.

Dent, E. W. and Kalil, K. (2001). Axon branching requires interactions between dynamic microtubules and actin filaments. J Neurosci 21, 9757-9769.

Draberova, E., Sulimenko, V., Vinopal, S., Sulimenko, T., Sladkova, V., D'Agostino, L., Sobol, M., Hozak, P., Kren, L., Katsetos, C. D., et al. (2017). Differential expression of human gamma-tubulin isotypes during neuronal development and oxidative stress points to a gamma-tubulin-2 prosurvival function. Faseb J 31, 1828-1846.

Ejaz, R., Lionel, A. C., Blaser, S., Walker, S., Scherer, S. W., Babul-Hirji, R., Marshall, C. R., Stavropoulos, D. J. and Chitayat, D. (2017). De novo pathogenic variant in TUBB2A presenting with arthrogryposis multiplex congenita, brain abnormalities, and severe developmental delay. Am J Med Genet A 173, 2725-2730. 
Ellis, P., Fagan, B. M., Magness, S. T., Hutton, S., Taranova, O., Hayashi, S., McMahon, A., Rao, M. and Pevny, L. (2004). SOX2, a persistent marker for multipotential neural stem cells derived from embryonic stem cells, the embryo or the adult. Dev Neurosci-Basel 26, $148-165$.

English, J. A., Dicker, P., Foecking, M., Dunn, M. J. and Cotter, D. R. (2009). 2-D DIGE analysis implicates cytoskeletal abnormalities in psychiatric disease. Proteomics $\mathbf{9}, 3368$ 3382.

Fame, R. M., MacDonald, J. L. and Macklis, J. D. (2011). Development specification, and diversity of callosal projection neurons. Trends Neurosci 34, 41-50.

Frotscher, M. (1998). Cajal-Retzius cells, Reelin, and the formation of layers. Curr Opin Neurobiol 8, 570-575.

Fujimoto, A., Enoki, H., Niimi, K., Nozaki, T., Baba, S., Shibamoto, I., Otsuki, Y. and Oanishi, T. (2021). Epilepsy in patients with focal cortical dysplasia may be associated with autism spectrum disorder. Epilepsy Behav 120:107990 doi: 10.1016/j.yebeh.2021.107990.

Gil-Sanz, C., Espinosa, A., Fregoso, S. P., Bluske, K. K., Cunningham, C. L., MartinezGaray, I., Zeng, H. K., Franco, S. J. and Muller, U. (2015). Lineage Tracing Using Cux2-Cre and Cux2-CreERT2 Mice. Neuron 86, 1091-1099.

Gil-Sanz, C., Franco, S. J., Martinez-Garay, I., Espinosa, A., Harkins-Perry, S. and Muller, U. (2013). Cajal-Retzius Cells Instruct Neuronal Migration by Coincidence Signaling between Secreted and Contact-Dependent Guidance Cues. Neuron 79, 461-477.

Go, C. D., Knight, J. D. R., Rajasekharan, A., Rathod, B., Hesketh, G. G., Abe, K. T., Youn, J. Y., Samavarchi-Tehrani, P., Zhang, H., Zhu, L. Y., et al. (2021). A proximitydependent biotinylation map of a human cell. Nature 595, 120-124.

Gonzalez, C., Canovas, J., Fresno, J., Couve, E., Court, F. A. and Couve, A. (2016). Axons provide the secretory machinery for trafficking of voltage-gated sodium channels in peripheral nerve. $P$ Natl Acad Sci USA 113, 1823-1828.

Gotz, M., Stoykova, A. and Gruss, P. (1998). Pax6 controls radial glia differentiation in the cerebral cortex. Neuron 21, 1031-1044.

Hartfuss, E., Galli, R., Heins, N. and Gotz, M. (2001). Characterization of CNS precursor subtypes and radial glia. Dev Biol 229, 15-30.

He, C. H., Zhang, L., Song, N. N., Mei, W. Y., Chen, J. Y., Hu, L., Zhang, Q., Wang, Y. B. and Ding, Y. Q. (2021). Satb2 Regulates EphA7 to Control Soma Spacing and SelfAvoidance of Cortical Pyramidal Neurons. Cereb Cortex, bhab321 doi: 10.1093/cercor/bhab321.

Hersheson, J., Mencacci, N. E., Davis, M., MacDonald, N., Trabzuni, D., Ryten, M., Pittman, A., Paudel, R., Kara, E., Fawcett, K., et al. (2013). Mutations in the autoregulatory domain of beta-tubulin 4a cause hereditary dystonia. Ann Neurol 73, 546553.

Hevner, R. F., Shi, L. M., Justice, N., Hsueh, Y. P., Sheng, M., Smiga, S., Bulfone, A., Goffinet, A. M., Campagnoni, A. T. and Rubenstein, J. L. R. (2001). Tbr1 regulates differentiation of the preplate and layer 6. Neuron 29, 353-366.

Hippenmeyer, S., Vrieseling, E., Sigrist, M., Portmann, T., Laengle, C., Ladle, D. R. and Arber, S. (2005). A developmental switch in the response of DRG neurons to ETS transcription factor signaling. Plos Biol 3, 878-890.

Hirokawa, N. and Takemura, R. (2004). Molecular motors in neuronal development, intracellular transport and diseases. Curr Opin Neurobiol 14, 564-573. 
Hirota, Y. and Nakajima, K. (2017). Control of Neuronal Migration and Aggregation by Reelin Signaling in the Developing Cerebral Cortex. Front Cell Dev Biol 5:40 doi 10.3389/fcell.2017.0040.

Hisaoka, T., Nakamura, Y., Senba, E. and Morikawa, Y. (2010). THE FORKHEAD TRANSCRIPTION FACTORS, Foxp1 AND Foxp2, IDENTIFY DIFFERENT SUBPOPULATIONS OF PROJECTION NEURONS IN THE MOUSE CEREBRAL CORTEX. Neuroscience 166, 551-563.

Hu, Y. Y., Sun, Q. W., Zhang, C., Sha, Q. Q. and Sun, X. L. (2015). RE1 silencing transcription factor (REST) negatively regulates ALL1-fused from chromosome 1q (AF1q) gene transcription. Bmc Mol Biol 16.

Ivanova, E. L., Gilet, J. G., Sulimenko, V., Duchon, A., Rudolf, G., Runge, K., Collins, S. C., Asselin, L., Broix, L., Drouot, N., et al. (2019). TUBG1 missense variants underlying cortical malformations disrupt neuronal locomotion and microtubule dynamics but not neurogenesis. Nat Commun 10:2129 doi: 10.1038/s41467-019-10081-8.

Jaglin, X. H., Poirier, K., Saillour, Y., Buhler, E., Tian, G. L., Bahi-Buisson, N., FalletBianco, C., Phan-Dinh-Tuy, F., Kong, X. P., Bomont, P., et al. (2009). Mutations in the beta-tubulin gene TUBB2B result in asymmetrical polymicrogyria. Nat Genet 41, 746-752.

Jamuar, S. S., Lam, A. T. N., Kircher, M., D'Gama, A. M., Wang, J., Barry, B. J., Zhang, X. C., Hill, R. S., Partlow, J. N., Rozzo, A., et al. (2014). Somatic Mutations in Cerebral Cortical Malformations. New Engl J Med 371, 733-743.

Jansen, A. C., Oostra, A., Desprechins, B., De Vlaeminck, Y., Verhelst, H., Regal, L., Verloo, P., Bockaert, N., Keymolen, K., Seneca, S., et al. (2011). TUBA1A mutations From isolated lissencephaly to familial polymicrogyria. Neurology 76, 988-992.

Keays, D. A., Tian, G., Poirier, K., Huang, G. J., Siebold, C., Cleak, J., Oliver, P. L., Fray, M., Harvey, R. J., Molnar, Z., et al. (2007). Mutations in alpha-tubulin cause abnormal neuronal migration in mice and lissencephaly in humans. Cell 128, 45-57.

Kennedy, M. J. and Ehlers, M. D. (2006). Organelles and trafficking machinery for postsynaptic plasticity. Annu Rev Neurosci 29, 325-362.

Kristiansen, L. V., Beneyto, M., Haroutunian, V. and Meador-Woodruff, J. H. (2006). Changes in NMDA receptor subunits and interacting PSD proteins in dorsolateral prefrontal and anterior cingulate cortex indicate abnormal regional expression in schizophrenia. Mol Psychiatr 11, 737-747.

Kumamoto, T., Toma, K., Gunadi, McKenna, W. L., Kasukawa, T., Katzman, S., Chen, B. and Hanashima, C. (2013). Foxg1 Coordinates the Switch from Nonradially to Radially Migrating Glutamatergic Subtypes in the Neocortex through Spatiotemporal Repression. Cell Rep 3, 931-945.

Kumar, R. A., Pilz, D. T., Babatz, T. D., Cushion, T. D., Harvey, K., Topf, M., Yates, L., Robb, S., Uyanik, G., Mancini, G. M. S., et al. (2010). TUBA1A mutations cause wide spectrum lissencephaly (smooth brain) and suggest that multiple neuronal migration pathways converge on alpha tubulins. Hum Mol Genet 19, 2817-2827.

Lai, M. M., Guo, Y., Ma, J., Yu, H. L., Zhao, D. D., Fan, W. Q., Ju, X. D., Sheikh, M. A., Malik, Y. S., Xiong, W. C., et al. (2015). Myosin X regulates neuronal radial migration through interacting with N-cadherin. Front Cell Neurosci 9:326 doi:

10.3389/fncel.2015.00326.

Lawrenson, I. D., Krebs, D. L., Linossi, E. M., Zhang, J. G., McLennan, T. J., Collin, C., Mcrae, H. M., Kolesnik, T. B., Koh, K., Britto, J. M., et al. (2017). Cortical Layer 
Inversion and Deregulation of Reelin Signaling in the Absence of SOCS6 and SOCS7. Cereb Cortex 27, 576-588.

Lee, H., Engel, U., Rusch, J., Scherrer, S., Sheard, K. and Van Vactor, D. (2004). The microtubule plus end tracking protein orbit/MAST/CLASP acts downstream of the tyrosine kinase Abl in mediating axon guidance. Neuron 42, 913-926.

Leone, D. P., Heavner, W. E., Ferenczi, E. A., Dobreva, G., Huguenard, J. R., Grosschedl, R. and McConnell, S. K. (2015). Satb2 Regulates the Differentiation of Both Callosal and Subcerebral Projection Neurons in the Developing Cerebral Cortex. Cereb Cortex 25, 3406-3419.

Li, D. Q., Hou, Y. F., Wu, J., Chen, Y., Lu, J. S., Di, G. H., Ou, Z. L., Shen, Z. Z., Ding, J. and Shao, Z. M. (2006). Gene expression profile analysis of an isogenic tumour metastasis model reveals a functional role for oncogene AF1Q in breast cancer metastasis. Eur J Cancer 42, 3274-3286.

Li, L., Jayabal, S., Ghorbani, M., Legault, L. M., McGraw, S., Watt, A. J. and Yang, X. J. (2019). ATAT1 regulates forebrain development and stress-induced tubulin hyperacetylation. Cell Mol Life Sci 76, 3621-3640.

Li, P., Ji, M., Lu, F., Zhang, J. R., Li, H. J., Cui, T. X., Wang, X. L., Tang, D. Q. and Ji, C. Y. (2014). Degradation of AF1Q by chaperone-mediated autophagy. Exp Cell Res 327, 48-56.

Li, W., Ji, M., Lu, F., Pang, Y. H., Dong, X., Zhang, J. R., Li, P., Ye, J. J., Zang, S. L., Ma, D. X., et al. (2018). Novel AF1q/MLLT11 favorably affects imatinib resistance and cell survival in chronic myeloid leukemia. Cell Death Dis 9(9):855 doi: 10.1038/s41419-0180900-7.

Lin, H. J., Shaffer, K. M., Sun, Z. R., Jay, G., He, W. W. and Ma, W. (2004). AF1q, a differentially expressed gene during neuronal differentiation, transforms HEK cells into neuron-like cells. Mol Brain Res 131, 126-130.

Lin, S. and Smith, G. M. (2015). Acetylation as a mechanism that regulates axonal regeneration. Neural Regen Res 10, 1034-1036.

Lopez-Bendito, G. and Molnar, Z. (2003). Thalamocortical development: How are we going to get there? (vol 4, pg 276, 2003). Nat Rev Neurosci 4, 422-422.

Marin, O. and Rubenstein, J. L. R. (2003). Cell migration in the forebrain. Annu Rev Neurosci 26, 441-483.

Mensen, V. T., Wierenga, L. M., van Dijk, S., Rijks, Y., Oranje, B., Mandl, R. C. W. and Durston, S. (2017). Development of cortical thickness and surface area in autism spectrum disorder. Neuroimage-Clin 13, 215-222.

Miyata, T., Kawaguchi, A., Okano, H. and Ogawa, M. (2001). Asymmetric inheritance of radial glial fibers by cortical neurons. Neuron 31, 727-741.

Moffat, J. J., Ka, M. H., Jung, E. M. and Kim, W. Y. (2015). Genes and brain malformations associated with abnormal neuron positioning. Mol Brain 8(1):72 doi: 1186/s13041=0150164-4.

Moon, H. M. and Wynshaw-Boris, A. (2013). Cytoskeleton in action: lissencephaly, a neuronal migration disorder. Wires Dev Biol 2, 229-245.

Mullen, R. J., Buck, C. R. and Smith, A. M. (1992). Neun, a Neuronal Specific NuclearProtein in Vertebrates. Development 116, 201-211.

Nadarajah, B., Brunstrom, J. E., Grutzendler, J., Wong, R. O. L. and Pearlman, A. L. (2001). Two modes of radial migration in early development of the cerebral cortex. Nat Neurosci 4, 143-150. 
Nance, J. and Zallen, J. A. (2011). Elaborating polarity: PAR proteins and the cytoskeleton. Development 138, 799-809.

Ngo, L., Haas, M., Qu, Z. D., Li, S. S., Zenker, J., Teng, K. S. L., Gunnersen, J. M., Breuss, M., Habgood, M., Keays, D. A., et al. (2014). TUBB5 and its disease-associated mutations influence the terminal differentiation and dendritic spine densities of cerebral cortical neurons. Hum Mol Genet 23, 5147-5158.

Nieto, M., Monuki, E. S., Tang, H., Imitola, J., Haubst, N., Khoury, S. J., Cunningham, J., Gotz, M. and Walsh, C. A. (2004). Expression of Cux-1 and Cux-2 in the subventricular zone and upper layers II-IV of the cerebral cortex. J Comp Neurol 479, 168-180.

Park, J., Hwang, J. Y., Thore, A., Kim, S., Togano, T., Hagiwara, S., Park, J. W. and Tse, W. (2019). AF1q inhibited T cell attachment to breast cancer cell by attenuating Intracellular Adhesion Molecule-1 expression. Journal of cancer metastasis and treatment 5:17 doi: 10.20517/2394-4722.2018.84.

Park, J., Schlederer, M., Schreiber, M., Ice, R., Merkel, O., Bilban, M., Hofbauer, S., Kim, S., Addison, J., Zou, J., et al. (2015). AF1q is a novel TCF7 co-factor which activates CD44 and promotes breast cancer metastasis. Oncotarget 6, 20697-20710.

Pennington, K., Beasley, C. L., Dicker, P., Fagan, A., English, J., Pariante, C. M., Wait, R., Dunn, M. J. and Cotter, D. R. (2008). Prominent synaptic and metabolic abnormalities revealed by proteomic analysis of the dorsolateral prefrontal cortex in schizophrenia and bipolar disorder. Mol Psychiatr 13, 1102-1117.

Piroli, M. E., Blanchette, J. O. and Jabbarzadeh, E. (2019). Polarity as a physiological modulator of cell function. Front Biosci-Landmrk 24, 451-462.

Poirier, K., Keays, D. A., Francis, F., Saillour, Y., Bahi, N., Manouvrier, S., Fallet-Bianco, C., Pasquier, L., Toutain, A., Tuy, F. P. D., et al. (2007). Large spectrum of lissencephaly and pachygyria phenotypes resulting from de novo missense mutations in tubulin alpha 1A (TUBA1A). Hum Mutat 28, 1055-1064.

Poirier, K., Lebrun, N., Broix, L., Tian, G. L., Saillour, Y., Boscheron, C., Parrini, E., Valence, S., Saint Pierre, B., Oger, M., et al. (2013). Mutations in TUBG1, DYNC1H1, KIF5C and KIF2A cause malformations of cortical development and microcephaly. Nat Genet 45, 962-962.

Poirier, K., Saillour, Y., Bahi-Buisson, N., Jaglin, X. H., Fallet-Bianco, C., Nabbout, R., Castelnau-Ptakhine, L., Roubertie, A., Attie-Bitach, T., Desguerre, I., et al. (2010). Mutations in the neuronal beta-tubulin subunit TUBB3 result in malformation of cortical development and neuronal migration defects. Hum Mol Genet 19, 4462-4473.

Reyes, N. S. D., Mederos, S., Varela, I., Weiss, L. A., Perea, G., Galazo, M. J. and Nieto, M. (2019). Transient callosal projections of L4 neurons are eliminated for the acquisition of local connectivity. Nat Commun 10(1):4549 doi: 10.1038/s41467=019-12495-w.

Rodan, L. H., El Achkar, C. M., Berry, G. T., Poduri, A., Prabhu, S. P., Yang, E. and Anselm, I. (2017). De Novo TUBB2A Variant Presenting With Anterior Temporal Pachygyria. J Child Neurol 32, 127-131.

Rodriguez-Tornos, F. M., Briz, C. G., Weiss, L. A., Sebastian-Serrano, A., Ares, S., Navarrete, M., Frangeul, L., Galazo, M., Jabaudon, D., Esteban, J. A., et al. (2016). Cux1 Enables Interhemispheric Connections of Layer II/III Neurons by Regulating Kv1Dependent Firing. Neuron 89, 494-506.

Ropars, V., Yang, Z. H., Isabet, T., Blanc, F., Zhou, K. F., Lin, T. M., Liu, X. Y., Hissier, P., Samazan, F., Amigues, B., et al. (2016). The myosin X motor is optimized for movement on actin bundles. Nat Commun 7:12456 doi: 10.1038/ncomms12456. 
Saito, T. (2006). In vivo electroporation in the embryonic mouse central nervous system. Nat Protoc 1, 1552-1558.

Sakakibara, A., Sato, T., Ando, R., Noguchi, N., Masaoka, M. and Miyata, T. (2014). Dynamics of Centrosome Translocation and Microtubule Organization in Neocortical Neurons during Distinct Modes of Polarization. Cereb Cortex 24, 1301-1310.

Schindelin, J., Arganda-Carreras, I., Frise, E., Kaynig, V., Longair, M., Pietzsch, T., Preibisch, S., Rueden, C., Saalfeld, S., Schmid, B., et al. (2012). Fiji: an open-source platform for biological-image analysis. Nat Methods 9, 676-682.

Sivagnanasundaram, S., Crossett, B., Dedova, I., Cordwell, S. and Matsumoto, I. (2007). Abnormal pathways in the genu of the corpus callosum in schizophrenia pathogenesis: a proteome study. Proteom Clin Appl 1, 1291-1305.

Stoner, R., Chow, M. L., Boyle, M. P., Sunkin, S. M., Mouton, P. R., Roy, S., WynshawBoris, A., Colamarino, S. A., Lein, E. S. and Courchesne, E. (2014). Patches of Disorganization in the Neocortex of Children with Autism. New Engl J Med 370, 12091219.

Sudo, H. and Baas, P. W. (2010). Acetylation of Microtubules Influences Their Sensitivity to Severing by Katanin in Neurons and Fibroblasts. J Neurosci 30, 7215-7226.

Thomson, A. M. (2010). Neocortical layer 6, a review. Front Neuroanat 4:13 doi: 10.3389/fnana.2010.00013.

Tian, G. L., Jaglin, X. H., Keays, D. A., Francis, F., Chelly, J. and Cowan, N. J. (2010). Disease-associated mutations in TUBA1A result in a spectrum of defects in the tubulin folding and heterodimer assembly pathway. Hum Mol Genet 19, 3599-3613.

Tiberio, P., Lozneanu, L., Angeloni, V., Cavadini, E., Pinciroli, P., Callari, M., Carcangiu, M. L., Lorusso, D., Raspagliesi, F., Pala, V., et al. (2017). Involvement of AF1q/MLLT11 in the progression of ovarian cancer. Oncotarget 8, 23246-23264.

Tischfield, M. A., Baris, H. N., Wu, C., Rudolph, G., Van Maldergem, L., He, W., Chan, W. M., Andrews, C., Demer, J. L., Robertson, R. L., et al. (2010). Human TUBB3 Mutations Perturb Microtubule Dynamics, Kinesin Interactions, and Axon Guidance. Cell 140, 74-87.

Toma, K., Kumamoto, T. and Hanashima, C. (2014). The Timing of Upper-Layer Neurogenesis Is Conferred by Sequential Derepression and Negative Feedback from Deep-Layer Neurons. J Neurosci 34, 13259-13276.

Tsai, J. W., Bremner, K. H. and Vallee, R. B. (2007). Dual subcellular roles for LIS1 and dynein in radial neuronal migration in live brain tissue. Nat Neurosci 10, 970-979.

Tse, C. O., Kim, S. and Park, J. (2017). Activation of Wnt signaling pathway by AF1q enriches stem-like population and enhance mammosphere formation of breast cells. Biochem Bioph Res Co 484, 884-889.

Tse, W., Meshinchi, S., Alonzo, T. A., Stirewalt, D. L., Gerbing, R. B., Woods, W. G., Appelbaum, F. R. and Radich, J. P. (2004). Elevated expression of the AFlq gene, an MLL fusion partner, is an independent adverse prognostic factor in pediatric acute myeloid leukemia. Blood 104, 3058-3063.

Tse, W., Zhu, W. M., Chen, H. S. and Cohen, A. (1995). A Novel Gene, Af1q, Fused to Mll in T(1-11)(Q21-Q23), Is Specifically Expressed in Leukemic and Immature HematopoieticCells. Blood 85, 650-656.

Tueting, P., Costa, E., Dwivedi, Y., Guidotti, A., Impagnatiello, F., Manev, R. and Pesold, C. (1999). The phenotypic characteristics of heterozygous reeler mouse. Neuroreport 10, 1329-1334. 
Wei, D., Gao, N. N., Li, L., Zhu, J. X., Diao, L., Huang, J. S., Han, Q. J., Wang, S. G., Xue, H. Q., Wang, Q., et al. (2018). alpha-Tubulin Acetylation Restricts Axon Overbranching by Dampening Microtubule Plus-End Dynamics in Neurons. Cereb Cortex 28, 33323346.

Weng, L. Q., Zhang, W. B., Ye, Y., Yin, P. P., Yuan, J., Wang, X. X., Kang, L., Jiang, S. S., You, J. Y., Wu, J., et al. (2014). Aliskiren ameliorates pressure overload-induced heart hypertrophy and fibrosis in mice. Acta Pharmacol Sin 35, 1005-1014.

Whitman, M. C., Andrews, C., Chan, W. M., Tischfield, M. A., Stasheff, S. F., Brancati, F., Ortiz-Gonzalez, X., Nuovo, S., Garaci, F., MacKinnon, S. E., et al. (2016). Two unique TUBB3 mutations cause both CFEOM3 and malformations of cortical development. Am J Med Genet A 170, 297-305.

Yamada, M., Clark, J. and Iulianella, A. (2014). MLLT11/AF1q is differentially expressed in maturing neurons during development. Gene Expr Patterns 15, 80-87.

Yamada, M., Clark, J., McClelland, C., Capaldo, E., Ray, A. and Iulianella, A. (2015). Cux2 Activity Defines a Subpopulation of Perinatal Neurogenic Progenitors in the Hippocampus. Hippocampus 25, 253-267.

Yao, F., Zhang, K. Y., Feng, C. Y., Gao, Y., Shen, L. M., Liu, X. K. and Ni, J. Z. (2021). Protein Biomarkers of Autism Spectrum Disorder Identified by Computational and Experimental Methods. Front Psychiatry 12:554621 doi: 10.3389/fpsyt.2021.554621.

Yuba-Kubo, A., Kubo, A., Hata, M. and Tsukita, S. (2005). Gene knockout analysis of two gamma-tubulin isoforms in mice. Dev Biol 282, 361-373.

Zhang, S. C. (2001). Defining glial cells during CNS development. Nat Rev Neurosci 2, 840843.

Zhou, F. Q., Zhou, J., Dedhar, S., Wu, Y. H. and Snider, W. D. (2004). NGF-induced axon growth is mediated by localized inactivation of GSK-30 and functions of the microtubule plus end binding protein APC. Neuron 42, 897-912.

Zimmer, C., Tiveron, M. C., Bodmer, R. and Cremer, H. (2004). Dynamics of Cux2 expression suggests that an early pool of SVZ precursors is fated to become upper cortical layer neurons. Cereb Cortex 14, 1408-1420. 
Figure 3: MIIt11 overexpression promoted migration into the cortical plate

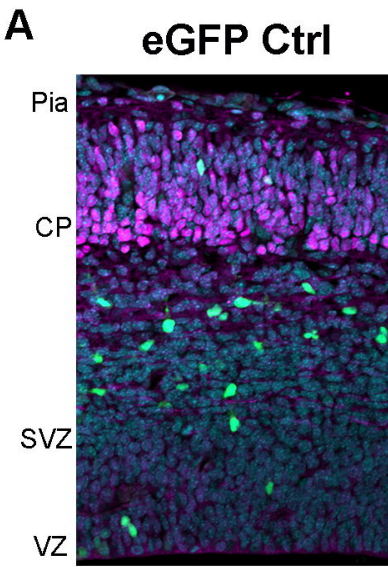

MIlt11-eGFP

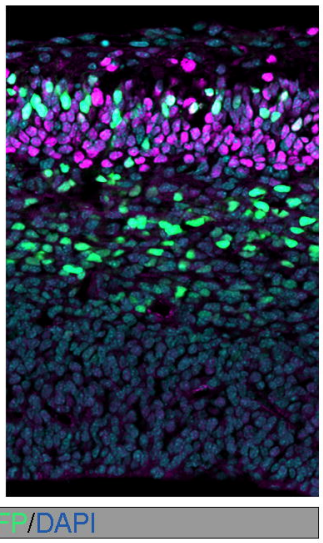

C

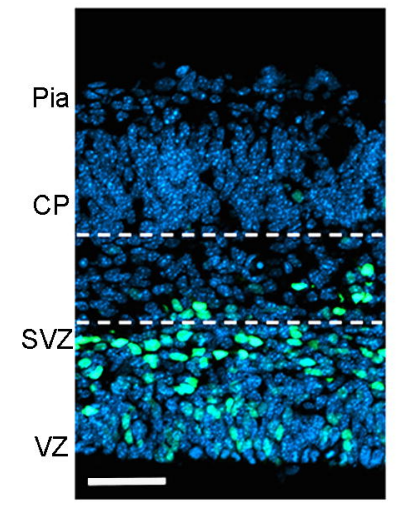

B

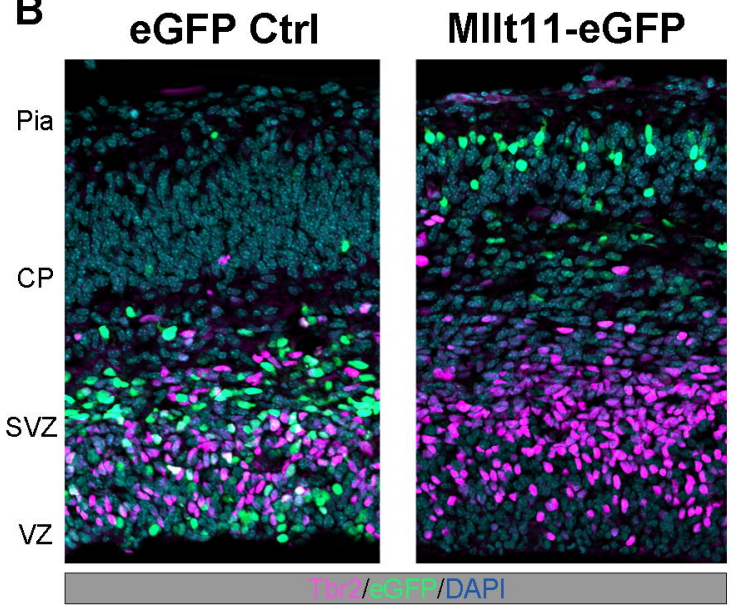

D Localization of electroporated cells

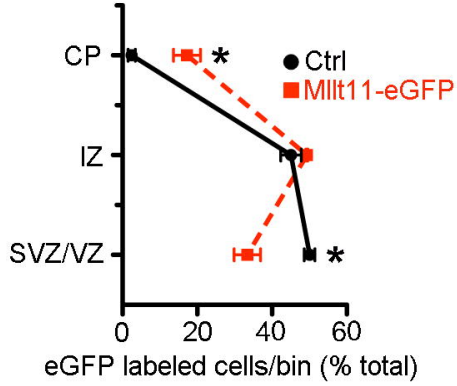


Figure 4: Formation of white matter tracts and callosal projections is impaired in the MIIt11 cKO cortex
A
B
C
D
E
$\mathbf{F}$
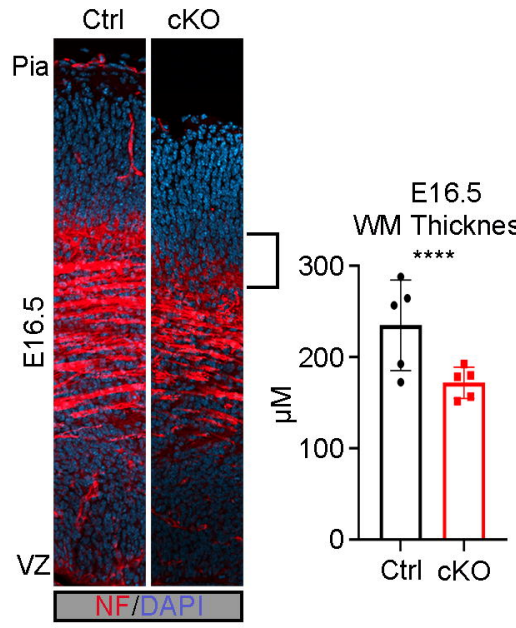

E16.5

WM as \% of Cortical

E18.5

WM Thickness

E18.5

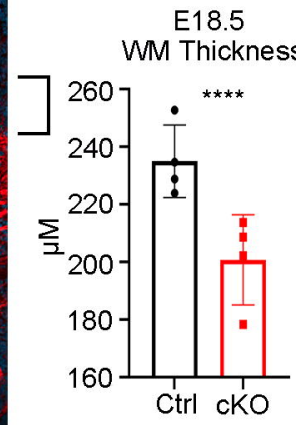

WM as \% of Cortical
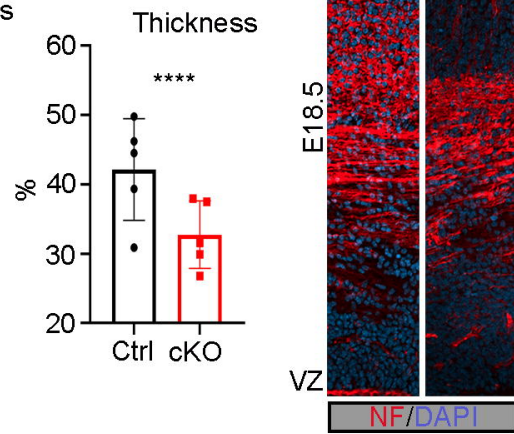

Thickness

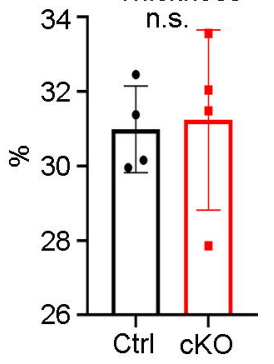

G

H

E18.5
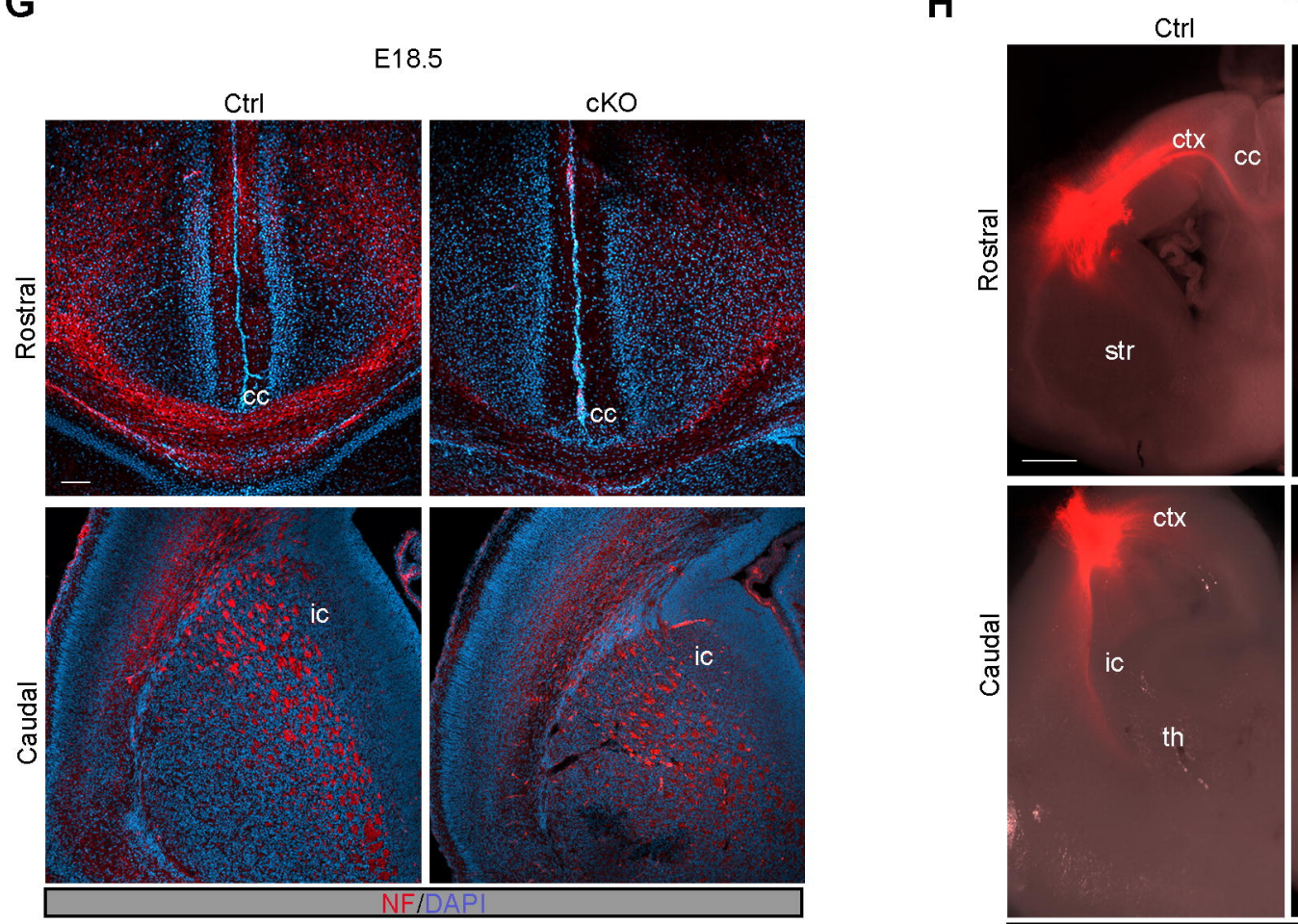

E18.5
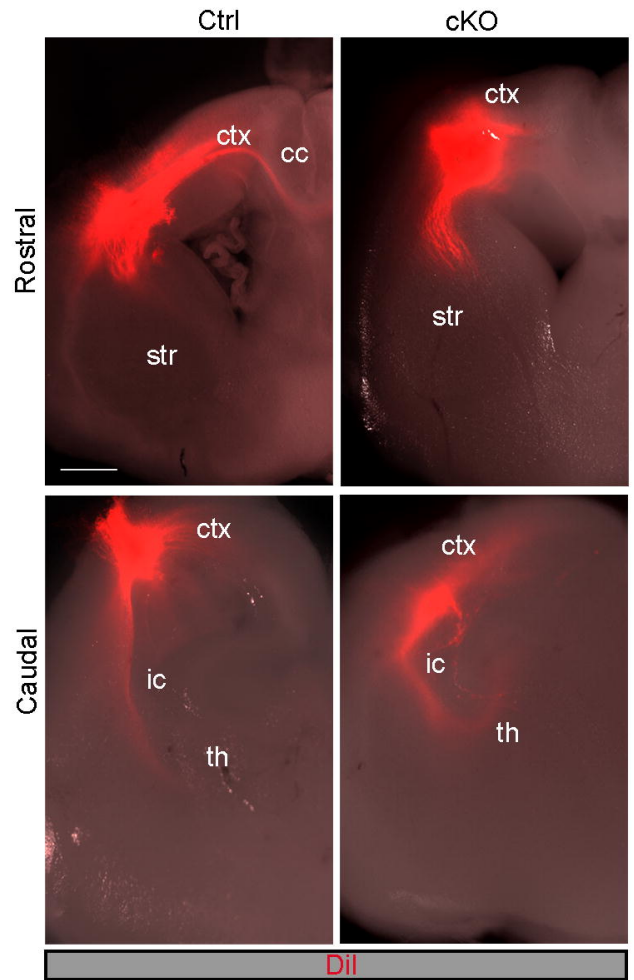

Figure 4 
Figure 5: Mllt11 associates and colocalizes with acetylated $\alpha$-tubulin
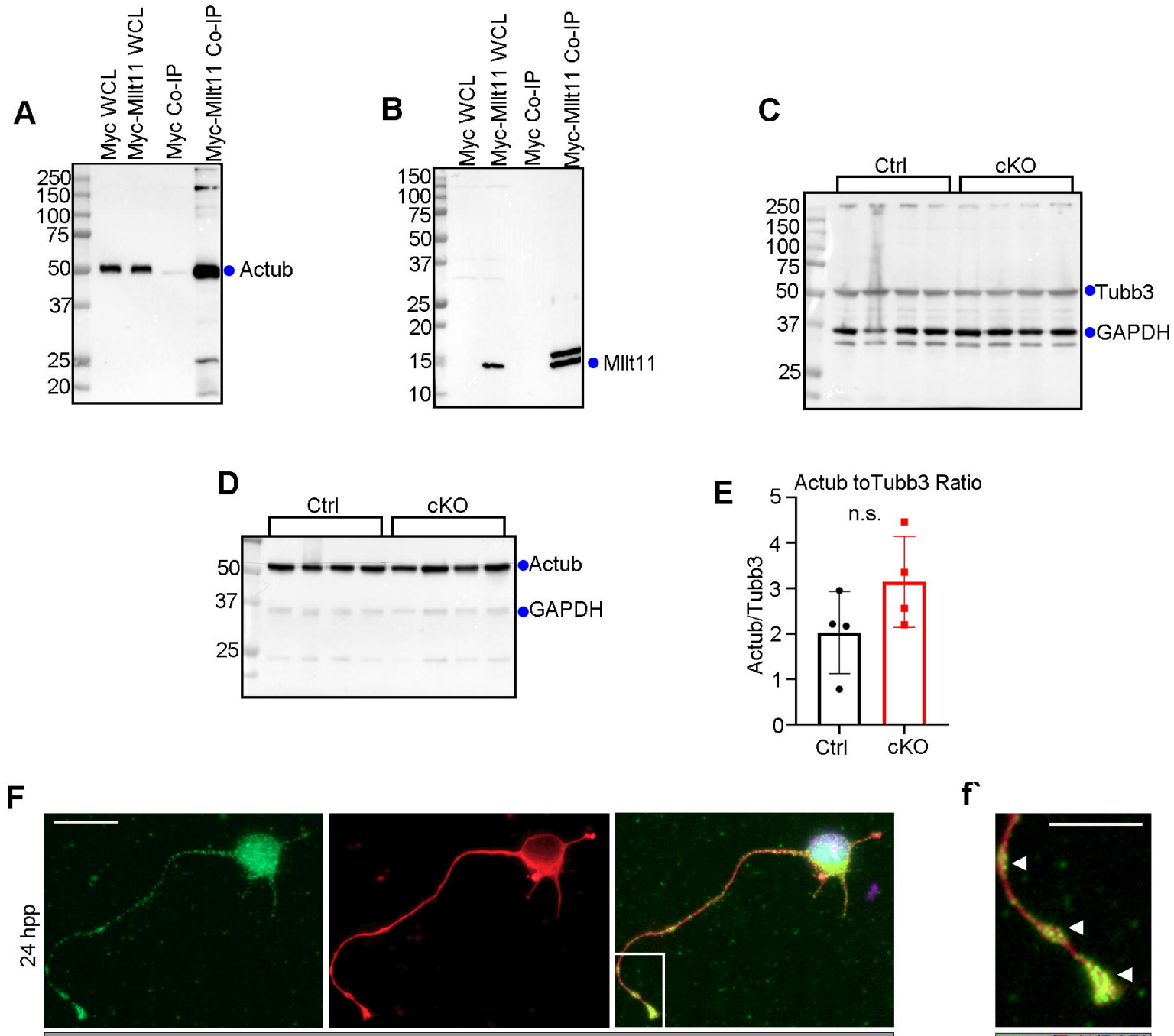

\section{f}
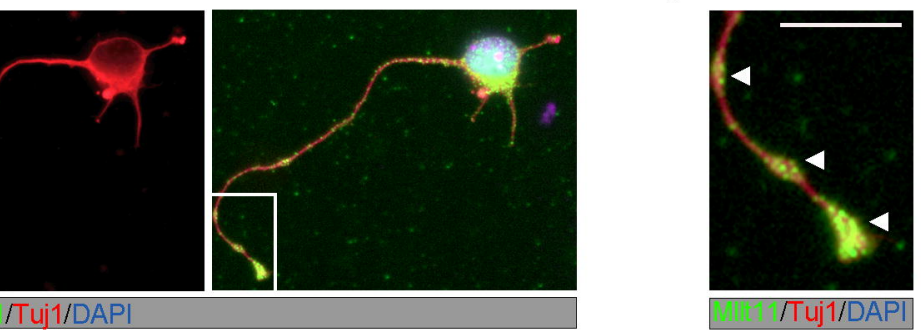

G

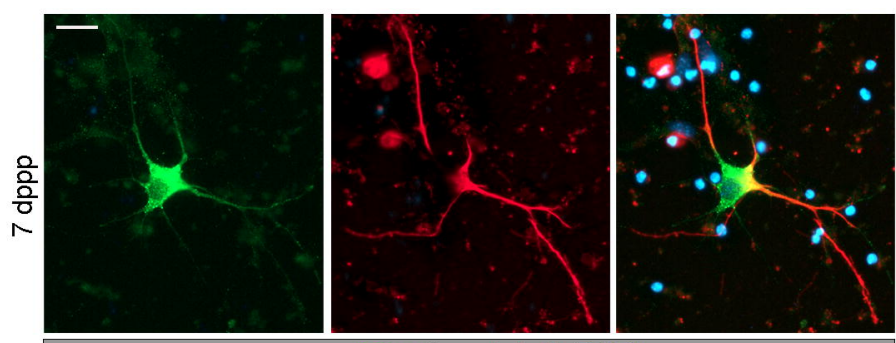


Figure 6: MIIt11 loss leads to decreased neurite outgrowth and branching complexity in vitro

A

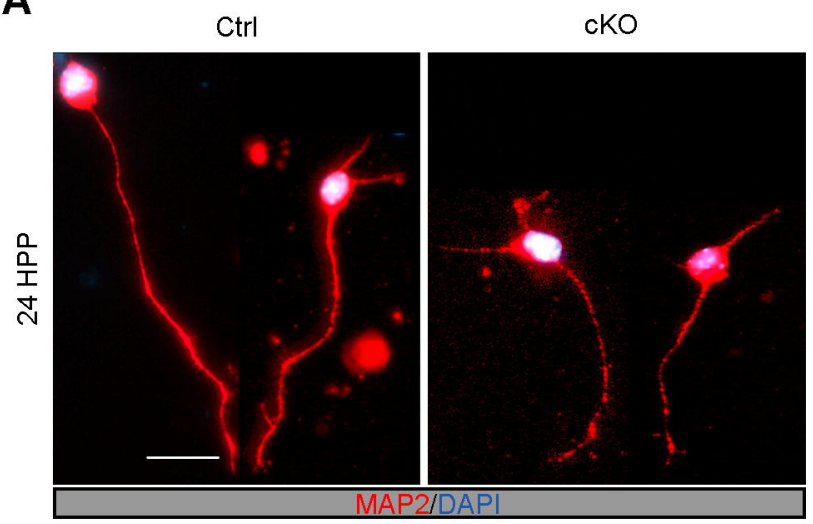

B

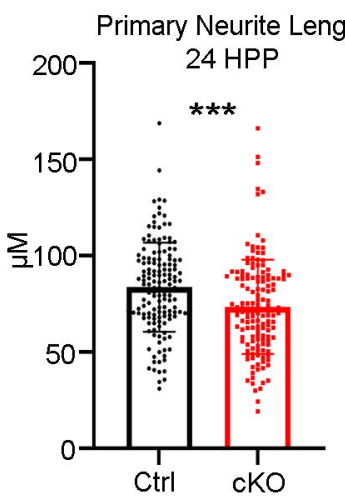

C

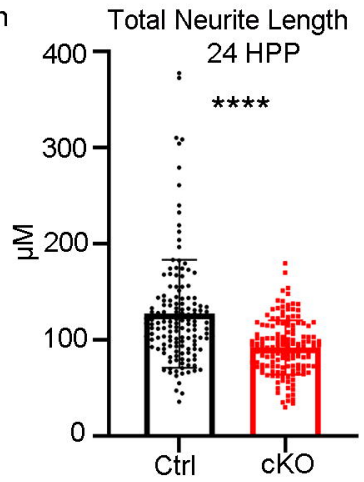

D Primary Neurite Length as Proportion of Total Neurite

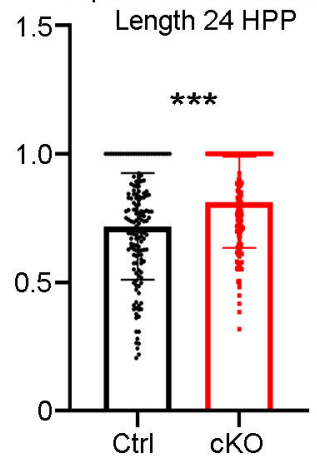

G

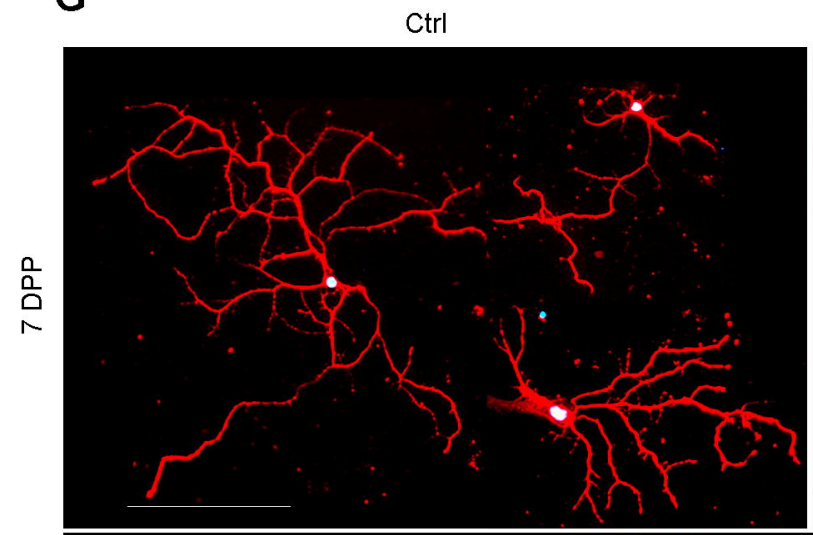

F

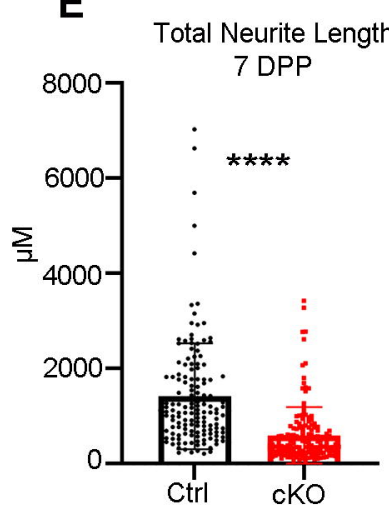

Sholl Analysis 7 DPP

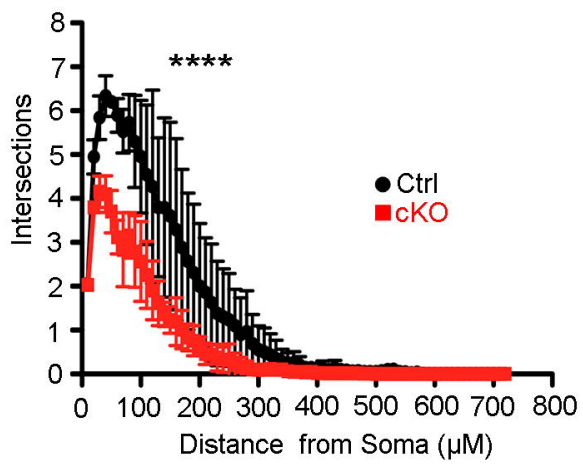

$\mathrm{cKO}$

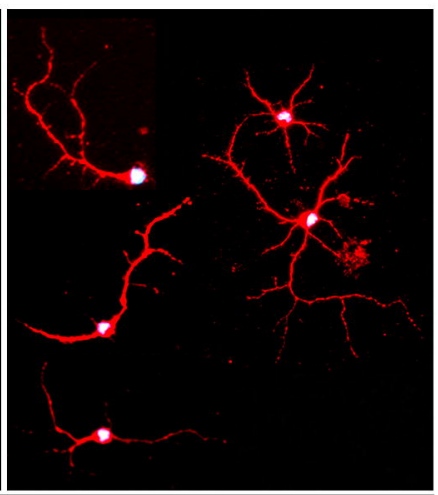


Figure 7: Decreased neurite outgrowth and branching complexity of UL CPNS in the MIIt11 cKO mutant cortex
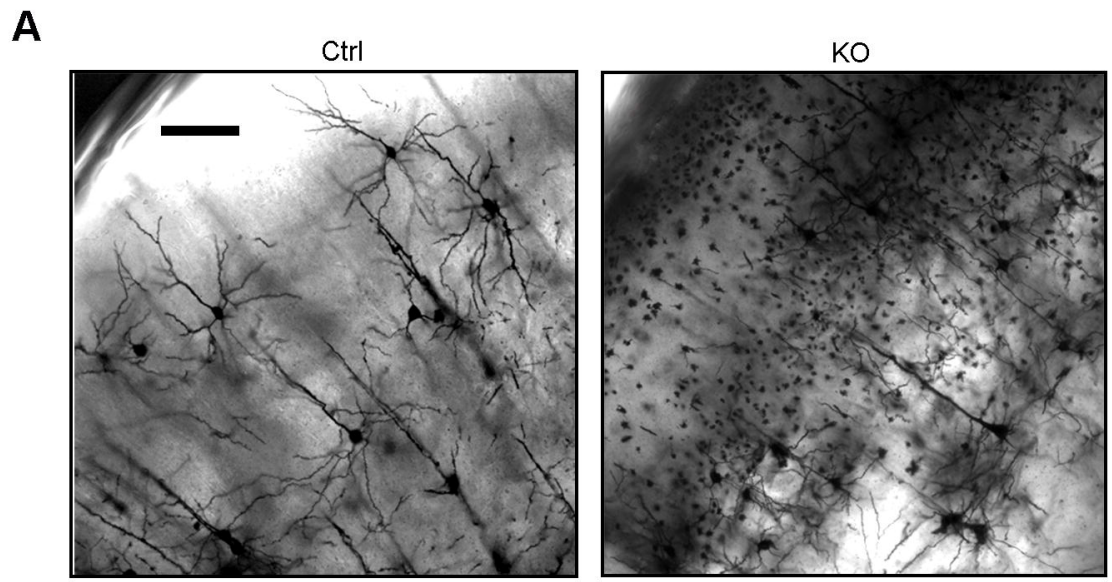

B
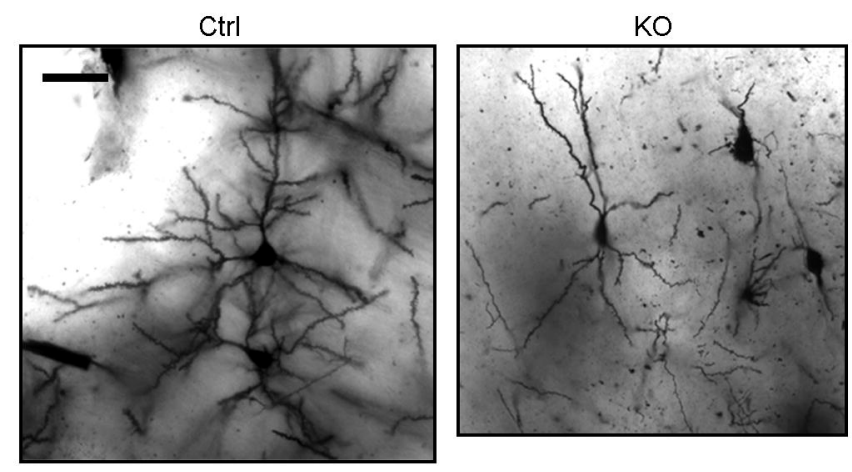

C
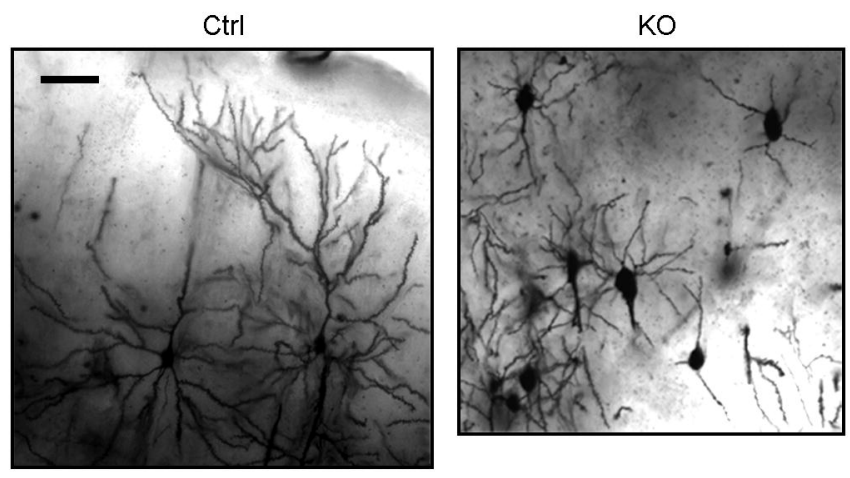

D

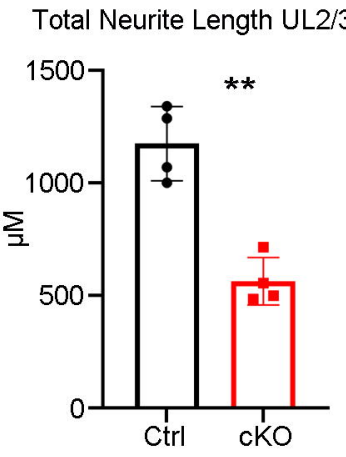

E

Sholl Analysis UL2/3

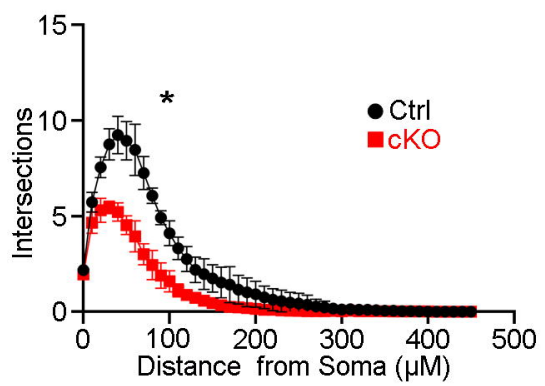

Figure 7 